\title{
Electromagnetic flanging: from elementary geometries to aeronautical components
}

\author{
Cheikh Tidiane Sow ${ }^{1}$, Grégoire Bazin ${ }^{2}$, Thomas Heuzé ${ }^{3}$, and Guillaume Racineux ${ }^{3}$ \\ ${ }^{1}$ IRT Jules Verne, Chemin du Chaffault, 44340 Bouguenais, France \\ ${ }^{2}$ STELIA Aerospace, 13 Boulevard des Apprentis, 44600 Saint-Nazaire, France \\ ${ }^{3}$ Research Institute in Civil and Mechanical Engineering (GeM, UMR 6183 CNRS), \\ Ecole Centrale de Nantes, 1 rue de la Noë, F-44321 Nantes, France
}

\begin{abstract}
Aeronautical companies are manufacturing many components of small (less than $200 \mathrm{~mm}$ ) and medium (between $200 \mathrm{~mm}$ and $1000 \mathrm{~mm}$ ) sizes by flexforming. In order to diversify the production facilities of these components, these companies are interested in evaluating the ability of electromagnetic forming processes to produce small parts. This paper describes the design of a set of experiments of electromagnetic flanging with some elementary geometries, whose purpose is to enlighten several geometrical defect issues encountered, and to propose some solutions. A particular attention is first paid to the straight flange, which allows to analyse, understand and then correct the main defect issues that can occur during the forming. The proposed solutions consist of some particular design of the inductors and the dies, in order to adjust the profile of the loading and the kinematics of bending of the flange to obtain the desired final geometry. Next, curved flanges are addressed, and finally the forming of a model aeronautical part combining several elementary geometries of flange is described. Promising results are obtained. In these experiments, sheets of 1,2 and $1.6 \mathrm{~mm}$ thickness made of aluminium alloy 2024-T4 and sheets of $0.5 \mathrm{~mm}$ thickness made of aluminium alloy 1050 are used. The geometric dispersions and the material soundness of the parts have been controlled. Besides, a numerical model of the model aeronautical part has been developed in the LS-DYNA computing environment, whose final purpose is to facilitate the design of the coil. The results of the numerical simulations are shown to be qualitatively in accordance with experimental results.
\end{abstract}

Keywords - Electromagnetic flanging, Aeronautical components, Geometrical defects, Optimized inductors

\section{Introduction}

The aeronautical industry traditionally manufactures many components made of lightweight alloys of small (less than $200 \mathrm{~mm}$ ) and medium (between 200 and $1000 \mathrm{~mm}$ ) sizes by flexforming [1], which consists in drawing sheet parts against an elastic matrix. In particular, the production of flanges of different shapes is widespread in aeronautics. However some aeronautical companies are interested in other processes for manufacturing these parts in order to diversify, improve their production means 
and to reduce costs. Indeed, improving the reproducibility of parts to reduce the production costs while still ensuring a high degree of geometrical precision and material soudness still remain a major challenge.

Exploiting velocity effects through high speed forming processes has been shown to offer different opportunities [2,3], among others the extension of the process limits for aluminum [4] or steel sheets [2], and can hence offer an alternative to more traditional processes used for aeronautical components. Indeed, the waves generated by dynamic loading yield a quite heterogeneous mechanical state in the structure, while materials submitted to high strain rates exhibit a completely different behavior compared to quasi-static situations [5]. Both items contribute to what are generally called 'velocity effects' whose benefits are generally observed on forming limits of metallic alloys classically reached with steady-state forming processes, that are dramatically increased at high strain rate. The gain is all the more significant that the alloy addressed has a low ductility at low strain rate. Hence, these processes have appeared to be promising to address issues like a lack of formability, wrinkling or spring back. Forming flanges of aeronautical components of small size may also benefit from high speed forming, which could contribute to improve cost-effectiveness, geometric dispersions while maintaining a high level of metallurgical quality of parts.

These 'velocity effects' can be implemented using high pulsed power technologies like Electromagnetic Forming (EF) [3], which turns out to be one of the easiest high speed forming method to implement. It allows to replace the mechanical action with contact of conventional technologies by a contact-free mechanical action arising from electrical effects. A capacitor bank charged to a fairly high voltage, discharges in few microseconds an oscillating current flowing through a coil in the vicinity of a workpiece. Eddy currents generated in the workpiece flow such that intense repulsive forces, the Lorentz forces, are generated which allow to deform the workpiece. Though that technology shares some features with explosive forming, it is easier to handle/industrialize and involve lower pressure levels while it still benefits from processing dynamically.

This work is concerned with the evaluation of the ability of electromagnetic forming to produce flanges for aeronautical components. Flanging $[6,7]$ is a forming operation that consists in bending a narrow strip at the edge of a metal sheet along a straight or curved line. The major difficulties to succeed in electromagnetic flanging is to master the geometrical tolerances of the formed part. Especially for straight flanges, the flatness and the straightness of the bent flanges is of particular concern once the forming operation is finished. These requirements implies that two major challenges must be met with the electromagnetic pulse technology: on the one hand a special electromagnetic inductor should be designed according to the geometry of the flange of interest. Its geometry should account for some desired spatial distribution of Lorentz forces, which should hence lead to a particular kinematics of bending of the flange. On the other hand, a dedicated die should also be designed, which will give the final geometry of the flange.

Electromagnetic flanging has already been studied with both experimental and numerical approaches, the latter being generally conducted using one of the available commercial finite element codes [8]. Previous studies have mainly focused on circular hole flanging [9, 10, 11, 12, 13], oblique hole flanging [14], or hemming [15], also few studies pertain to straight flanges [16, 17, 18]. Results obtained from electromagnetic flanging appear promising, although the process remains to be optimized and some phenomena explained. For instance, the effect of specimen planar area [18] or the appearance of defects like non-straightness of flanges [17] have been reported, which depend on the adequacy between the chosen inductor and specimen geometries. In addition, a rebound of the flange after its contact with the die was reported [10,11, 19, 20], and analyzed both experimentally and numerically. Different propositions have been made to reduce this rebound. A first solution consists 
in introducing an appropriate die compensation angle [10] in the geometry of the die, although a particular compromise between the die compensation angle and the discharge energy has to be observed to reduce the flanging angle deviation and obtain a successful flanging. A second solution introduces a cushion (or driver or damping) plate [19], which can be added an intermediate elastic medium [20], that plays the role of a pusher and allows to reduce the magnitude of the rebound.

This paper describes the design of a set of experiments of electromagnetic flanging, whose purpose is (i) to enlighten issues related to the occurrence of some geometrical defects encountered in the formed flange, (ii) propose some physically based explanations and (iii) propose some solutions to address these issues. Four defect issues are considered in this work: (i) the so-called "edge effect", somewhat related to the "specimen planar area" [18], (ii) the formation of plastic hinges in the flange, (iii) the rebound between the flange and the die, and (iv) the formation of wrinkles in some curved flanges. Although hundreds of references of small flanged parts are available in the aeronautical industry, they are all made up of a limited set of elementary geometries. The elementary geometries considered in this work are straight, convex and concave flanges as well as circular hole and jogged flanges. Sheets of 1,2 and $1.6 \mathrm{~mm}$ thickness made of aluminium alloy 2024-T4 and sheets of thickness equal to $0.5 \mathrm{~mm}$ made of AA1050 will be used in this work.

A particular attention is first paid to the straight flange, which is the simplest geometry to be first considered for analysing, understanding and then correct the main defect issues occuring during the forming operation. The use of fast cameras enables us to observe the kinematics of bending of the flange, identify areas where Lorentz forces are too weak, and observe rebounds with the die. The proposed solutions consist of some particular design of the inductors and the dies, in order to adjust the profile of the loading and the kinematics of bending of the flange to obtain the desired final geometry. The geometric dispersions and the material soundness of the parts have been controlled, and satisfy the aeronautical requirements. A simple one-dimensional analytical modeling is described, which allows to explain qualitatively the occurrence of a rebound between the flange and the die. Next, curved flanges are addressed. The importance of a particular kinematics that consists in unrolling the flange onto the die is emphasized, which should be respected to perform a successful curved flange. Finally, electromagnetic flanging of a model aeronautical part combining several elementary geometries of flanges is described. A numerical model of that part has been developed in the LSDYNA computing environment [8], whose final purpose is to facilitate the design of the coil. The results of the numerical simulations are shown to be qualitatively in accordance with experimental results, although the combination of several elementary geometries of flanging in one part raises the question of the optimization of the geometries of the inductor and the die, due to the appearance of spurious electromagnetic interactions generated in that case.

The outline of the paper is as follows. First, the experimental material is presented in Section 2. The pratical implementation of electromagnetic flanging is presented, especially through the developed experimental setups and the instrumentation. Next, the methodology followed in the experimental analysis in presented in Section 3. Experimental results are then presented in Section 4, successively for the different experiments conducted. Discussions and analyses about the appearance of some defects and the solutions proposed are also included. Section 5 presents a simplified onedimensional modeling of the rebound. Finally, Section 6 is devoted to results obtained for a model aeronautical part, including experimental and numerical results, as well as a comparison between both. 


\section{Experimental material}

\subsection{Electromagnetic flanging}

Electromagnetic flanging consists in bending a narrow strip at the edge of a thin metal sheet along a straight or curved line, by means of a high discharged oscillating current flowing through a coil, which is placed in the vicinity of the narrow strip to be bent (see Figure 1(b)).

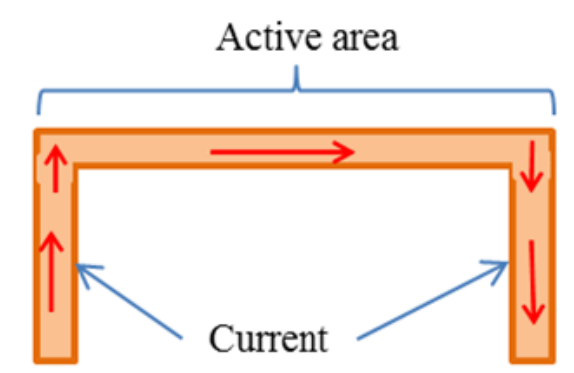

(a)

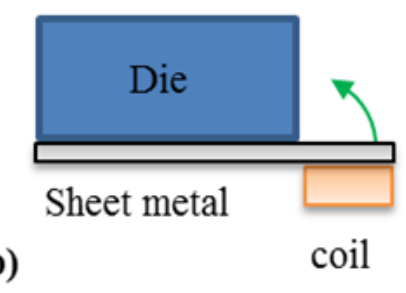

Figure 1: a) U-shaped geometry of the coil, b) action of the coil.

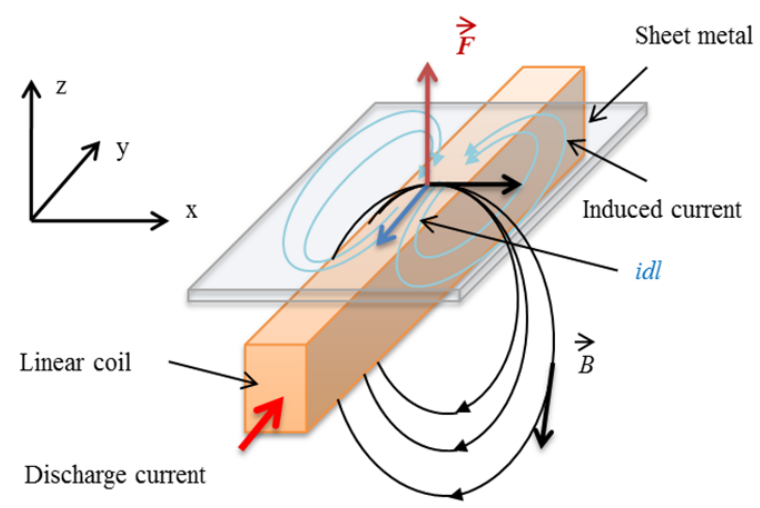

Figure 2: Sketch of eddy currents and magnetic streamlines around a linear inductor.

Basically for a straight flange, a U-shaped inductor (Figure 1(a)) consists of bars that supply the electrical power, and of a distributor of eddy currents. As the discharged current flows in the "active area" (Figure 2), eddy currents are induced within the flat sheet placed above, so that Lorentz forces are generated whose normal component (in the $z$ direction) is a combination of in-plane components of the magnetic induction $\mathbf{B}$ and the current density $\mathbf{j}$ vectors:

$$
F_{z}=j_{x} B_{y}-j_{y} B_{x}
$$

but essentially induced by the tangential component $B_{x}$ of the magnetic induction, the first term being neglectable in that case. Considering a straight flange as shown in Figure 3, a distinction will be made in this paper between the corners, the end and the center of a flange along its longitudinal direction $(y$-direction), in which Lorentz forces are expected to be of different magnitude due to geometrical effects. The top and base areas of the flange, along the transverse direction, will also be 


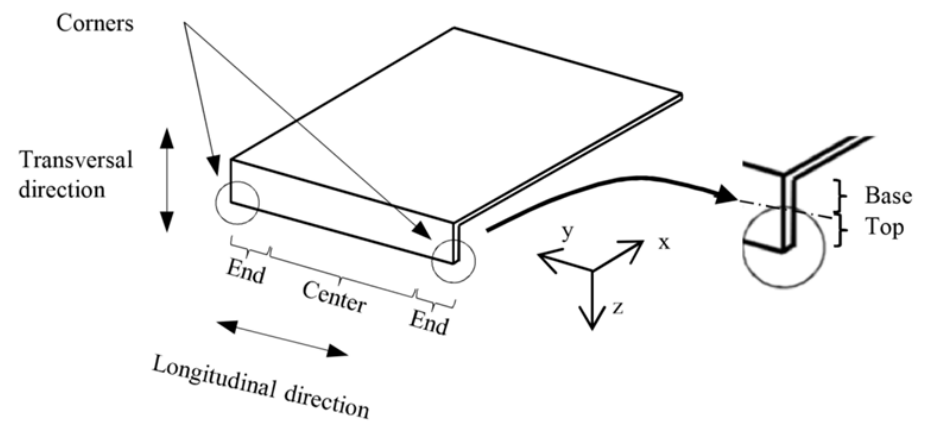

Figure 3: Definition of the vocabulary for a straight flange.

of pratical use. The transverse direction of the flange is expected to be aligned with the normal of the sheet $(z$-direction) once bent at a right angle.

\subsection{Experimental setup}

The experimental setup first consists of a capacitor bank of $46 \mathrm{~kJ}$, whose technical properties are listed in Table 1. Next, although hundreds of references of flanged parts are available in the aeronautical

\begin{tabular}{|l|c|}
\hline Charge voltage $(\mathrm{kV})$ & 15 \\
Capacitance $(\mu \mathrm{F})$ & 408 \\
Inductance $(\mu \mathrm{H})$ & 0.1 \\
Resistance $(\mathrm{m} \Omega)$ & 14 \\
Maximum current $(\mathrm{kA})$ & 500 \\
\hline
\end{tabular}

Table 1: Technical properties of the $46 \mathrm{~kJ}$ generator.

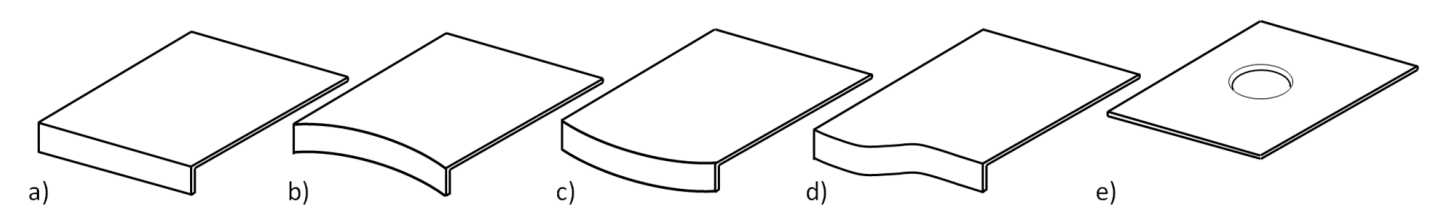

Figure 4: Generic geometrical configurations for flanging: a) straight flange, b) concave flange, c) convex flange, d) jogged flange, e) circular hole flange.

industry, they are all made up of a limited set of elementary geometries. Figure 4 shows some examples of elementary geometries considered here: straight, concave, convex, jogged and circular hole flanges. The sheets used for the experiments are made of aluminium alloy 2024-T4, whose chemical composition and quasi-static mechanical properties are listed in Tables 2 and 3 respectively. 


\begin{tabular}{|l|l|l|l|l|l|l|l|l|l|l|}
\hline Alloy & $\mathrm{Al}$ & $\mathrm{Si}$ & $\mathrm{Fe}$ & $\mathrm{Cu}$ & $\mathrm{Mn}$ & $\mathrm{Mg}$ & $\mathrm{Cr}$ & $\mathrm{Zn}$ & $\mathrm{Ti}$ & $\mathrm{Ga}$ \\
\hline 2024-T4 & 98.44 & 0.03 & 0.05 & 4.45 & 0.43 & 1.49 & 0.03 & 0.05 & 0.02 & 0.01 \\
\hline
\end{tabular}

Table 2: Chemical composition of the 2024-T4 aluminium alloy used (\% by mass).

\begin{tabular}{|c|c|c|c|}
\hline Direction & $R_{m}(\mathrm{MPa})$ & $R_{p_{0.2}}(\mathrm{MPa})$ & $\mathrm{A} 50(\%)$ \\
\hline $\mathrm{L}$ & 445 & 325 & 18 \\
\hline $\mathrm{T}$ & 435 & 300 & 18 \\
\hline
\end{tabular}

Table 3: Quasi-static mechanical properties of the 2024-T4.

The sheets have a thickness of 1,2 and $1.6 \mathrm{~mm}$. Sheets of thickness equal to $0.5 \mathrm{~mm}$ made of commercially pur aluminium 1050 will also be used for some tests for its good compliance and low threshold, in order to better observe geometrical defects.

Next, the setup consists of inductors. A U-shaped inductor is used, as well as optimized ones whose geometry for a straight flange is depicted in Figure 5. Optimized inductors still consist of

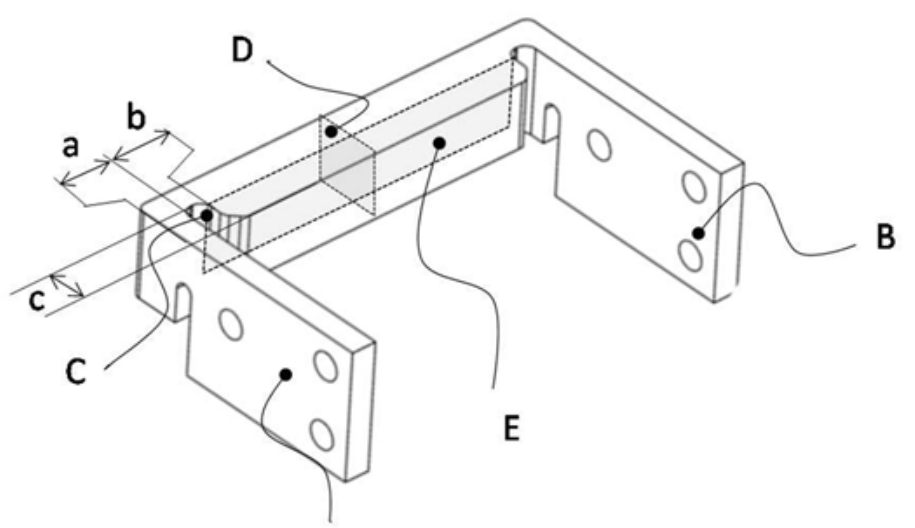

A

Figure 5: Principle of optimized inductors. A,B: power bars, C: notch, D: transversal cross-section, E: longitudinal cross-section.

power bars $(\mathrm{A}, \mathrm{B})$ supplying the electrical power and of a distributor of eddy currents, but notches (C) are machined close to the two corners of the flange, whose dimensions are denoted $a, b$ and $c$. Besides, the height of their active area (in the transverse direction, see Figure 3 ) is equal to that of flanges. These inductors are made of steel to increase their strength.

Such optimized notched inductors are designed for each elementary geometry of flange considered in this work (Figure 4). These are shown in Figure 6 for the straight, concave, convex and circular hole flanges. For the three first ones, the principle essentially amounts to bend the previous inductor in the right locations, according to the geometry of flanges. Notice that the thickness (in the $z$ direction) of the cross-section (D) of the optimized inductors designed for curved flanges may not be 
constant. A particular evolution of the thickness with the curvilinear coordinate may be of pratical interest to enforce some desired kinematics of the forming of the curved flange. The inductor for the circular hole flange, shown in Figure 6d), does not require any additional notches. But it can be seen that the input and output power bars are designed such that they are not adjacent to each other, meaning that the discharged current in these bars flow in the same direction. This design is desirable to avoid the occurrence of defects on the flange in the vicinity of input and output bars. Detailed explanations are provided at the end of Section 4.

The design of these inductors has been performed based on a technological know-how in mechanical and electromagnetic forming engineering by means of a trial and error-based process. More details and explanations about their design will be provided in Section 4, and in particular in Section 4.2 for curved inductors.

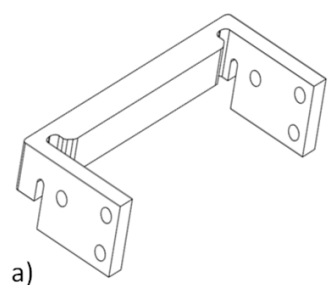

b)

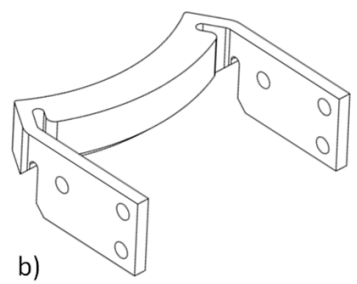

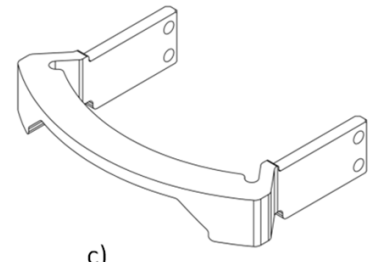

c)

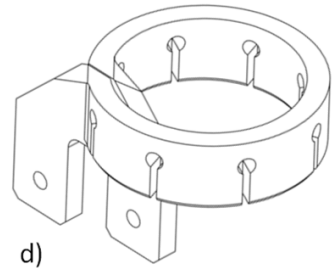

d)

Figure 6: Inductors for a) straight, b) concave, c) convex and d) circular hole flanges.

The setup next consists of dies, made of steel or aluminium 7075-0 depending on the experiment carried out, whose geometries are adapted to each elementary geometry of flange. Figure 7 shows assemblies consisting of supports, optimized inductors, sheets and dies for straight, jogged, concave, convex and circular hole flanges. Note also that a die compensation angle is machined in each die, in order to compensate the rebound generated after the flange/die impact. Die compensation angles of 9 and 7 degrees are respectively chosen for sheets of thickness 1 and $2 \mathrm{~mm}$.

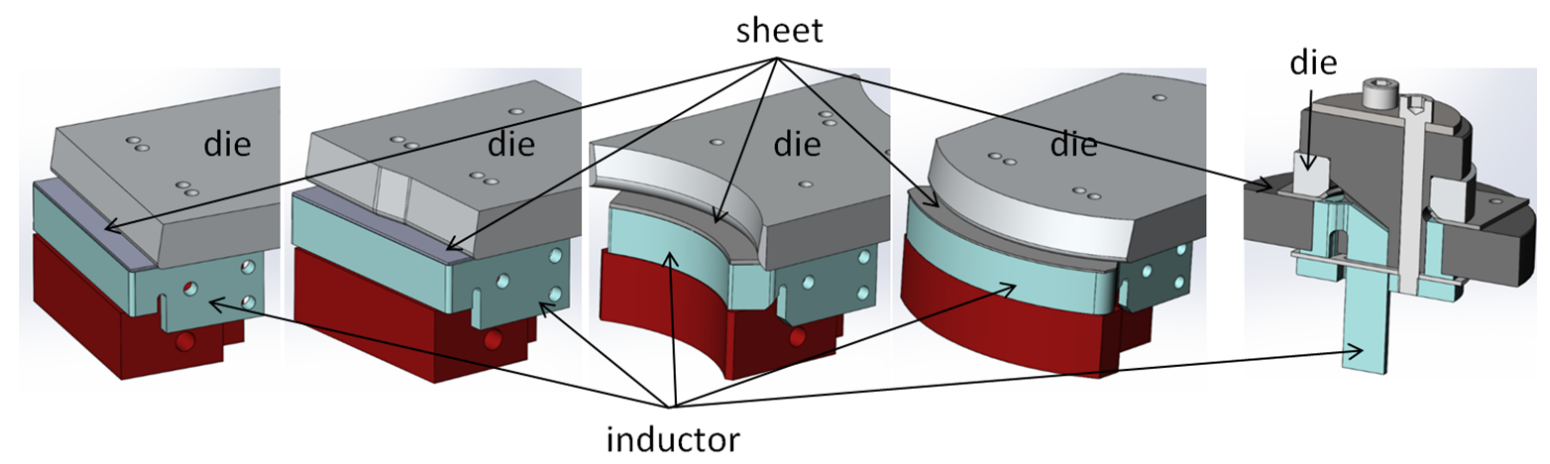

Figure 7: Geometries of the dies.

The setup finally consists of positioning and clamping systems. The positioning system is shown 

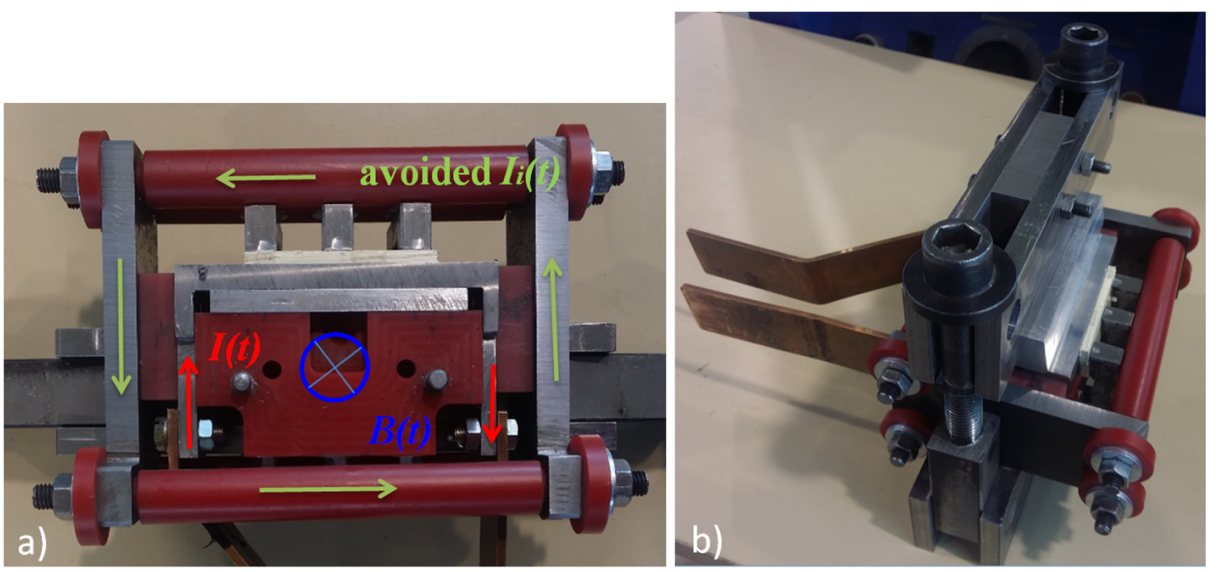

Figure 8: Clamping system.

in assemblies in Figure 7, and consists of a support made of polyurethane with pins, that allows reproducibility of positioning aluminium sheets with respect to inductors. Since inductors are submitted to very high loadings, the clamping system is here to constraint it in order to avoid any permanent deformation. In addition, the input oscillating current $I(t)$ flowing in the inductor, as shown in Figure 8 , generates a time-varying magnetic induction $B(t)$. The latter can potentially induce eddy currents $I_{i}(t)$ in the clamping device if it is not sufficiently isolated, generating a high mutual inductance and hence dropping the overall efficiency of the setup. Thus, thin-walled tubes of polyurethane are used to isolate threaded rods, washers and nuts are also isolated.

\subsection{Instrumentation and means of analysis}

Fast cameras Photron SA1 are used to film the forming process. Top and side views are retained to correctly observe the kinematics of forming of flanges. Rogowski coils are used to measure the discharged currents. Metrological controls are performed though 3D measurements. At last, metallographic analysis have been carried out through polishing and anodic oxidation.

\subsection{Experimental setup for a model aeronautical part}

The main challenge to build a typical part for the aeronautical industry pertains to the combination of some of the previous elementary geometries, because the expected flow of eddy currents for each elementary geometry can be disturbed by the combination of several of these. The next step consists in combining the previous geometries of inductors as subsets of a global inductor for the typical part, and in optimizing the geometry of this inductor in order to better account for both eddy currents in the workpiece and self-eddy currents interactions in the inductor. Figure 9 (a) shows the geometry of a model aeronautical part, and Figure 9(b) shows a global view of the assembly of the experimental setup. This part consists of two straight flanges which are not orthogonal, one jogged flange and one circular hole flange, so that no plane or axis of symmetry exist. Particular inductors and a dedicated setup have been designed, although their details are not shown here since they remain the property 


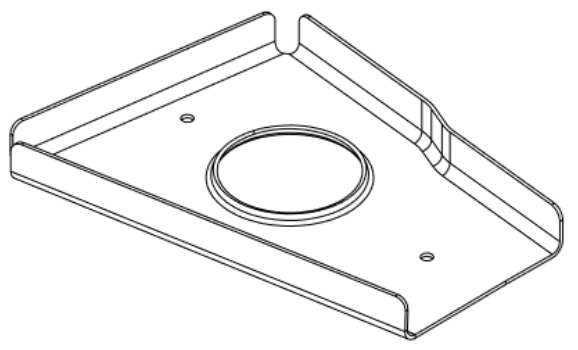

(a) Geometry

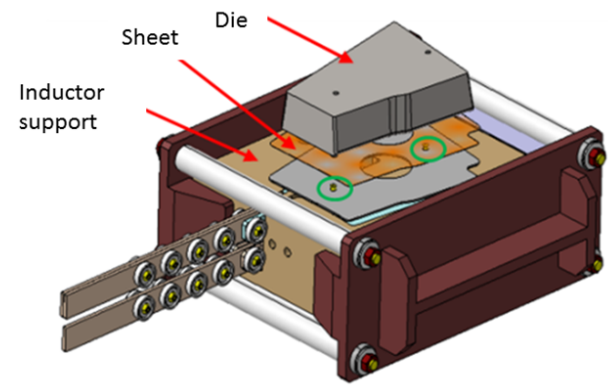

(b) Experimental setup

Figure 9: Model aeronautical part.

of the industrial. The forming operation is run in two steps: a first inductor allows the forming of the circular hole flange, then a second one permits the simultaneous forming of the two straight flanges and the jogged flange. This setup keeps the basic features already detailed for elementary geometries.

\section{Methodology}

Three types of experiments have first been conducted with different inductors for the straight flanges.

1. The purpose of the first experiment is to observe the occurrence of side effects. To this end, a U-shaped inductor is used (Figure 1a)), whose length of the active area nearly equals that of the flange. Aluminium alloy 1050 is used here in order to better observe the defects obtained with electromagnetic flanging, since this alloy is very compliant with a small yield threshold. Sheets of thickness $t=0.5 \mathrm{~mm}$, of flange height $H=10 \mathrm{~mm}$, of flange length $L=100 \mathrm{~mm}$, and of inner radius of folding $R_{i}=2.5 \mathrm{~mm}$ are used. Furthermore, dies made of steel are considered. The discharged energy for this experiment is about $1.8 \mathrm{~kJ}$.

2. The second experiment uses the inductor shown in Figure 5, whose purpose is to optimize the spatial distribution of Lorentz forces in the longitudinal direction. These experiments have been conducted with aluminium sheets AA2024-T4 of thickness 1 and $2 \mathrm{~mm}$ with the same flange height, for which discharged energies of 5.5 and $14 \mathrm{~kJ}$ have been chosen, corresponding to maximum discharged currents of about 200 and $330 \mathrm{kA}$. A minimum of ten tests of repeatability have been carried out for each thickness. Metrological controls have been carried out to check the obtained tolerances and deviations, and metallographic analyses have also been performed.

3. The third experiment investigates the influence of the flange height on its bending kinematics. The flange height is raised to $20 \mathrm{~mm}$ to show significant differences. The purpose is to address the formation of additional plastic hinges, that is the formation of additional section of the flange where plastic bending occurs. Some modifications are made to the geometry of the inductor to improve the spatial distribution of Lorentz forces in the transverse direction, while the discharged energies remain unchanged. 
Next, experiments for curved flanges have been carried out. Sheets made of AA2024-T4 of 1 and $2 \mathrm{~mm}$ thickness have been used, with discharged energies of 6.5 and $12 \mathrm{~kJ}$. Wrinkling and buckling phenomena are addressed in order to get a satisfactory flange.

At last, results obtained for a model aeronautical part are shown in Section 6. This part is formed from $1.6 \mathrm{~mm}$ sheet thickness, both Aluminium Alloy 2024-T4 and 1050 are used. Discharge energies of 6 and $10 \mathrm{~kJ}$ are used for the first and second coil respectively. Results obtained from a numerical model of that part are also shown, and a comparison between experimental and numerical results is performed.

\section{Results and discussions}

\subsection{Straight flanges}

\subsubsection{First experiment}

Results Figure 10 shows a sequence of pictures obtained with the high speed cameras, in top and side views recorded at 52000 frames per seconds. The top view allows to perform the following

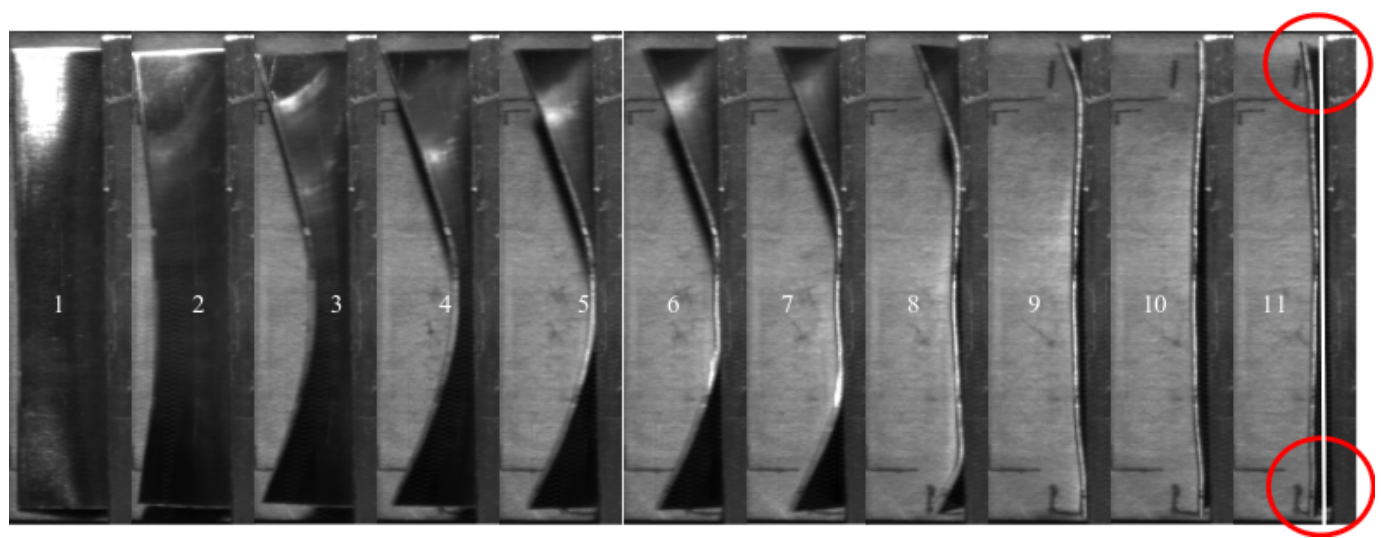

(a) Top view of the deformation process

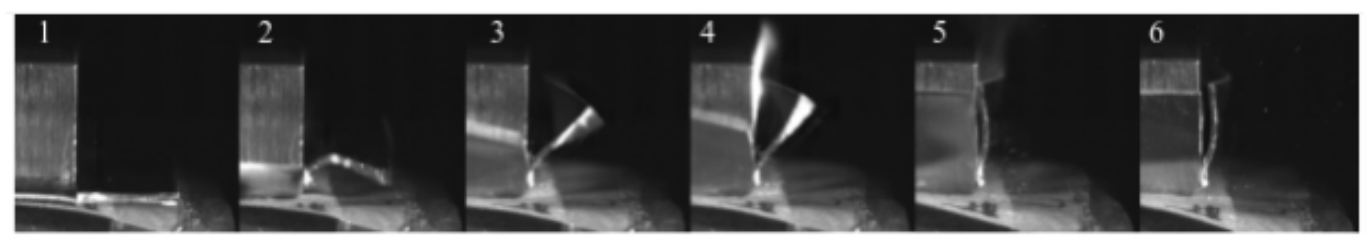

(b) Side view of the deformation process

Figure 10: Top and side views of forming a straight flange with high speed camera (52000 frames per seconds).

observations: 
(i) the two end extemities of the flange are late compared to its center, and bending of the flange also occurs about the transverse direction. Consequently, the contact of these extremities with the die is delayed.

(ii) the central part of the flange first impacts the die (picture 5) and then separates from the die before the two delayed corners contact the die (picture 10). Then, these corners separate (picture 11), and a significant amount of elastic bending oscillations are required to reach the equilibrium state. The flanging is finished in about $130 \mu \mathrm{s}$.

Next, the side view allows to observe that the flange is not bent in a uniform manner along its transverse direction, about the longitudinal one. Indeed, the formation of a plastic hinge can be observed in picture 2. The top of the flange then catches up with its base, and impacts the die first, where a flash is observed (picture 4). Finally, the obtained flange exhibits significant defects of flatness, straightness and perpendicularity with respect to the sheet (picture 6).

Discussion The two end extemities of the flange are late with respect to its center due to locally lower Lorentz forces. The straight coil (or U-shaped inductor) does not induce sufficient Lorentz forces in the corner of the flange, so that its extremities also rotate properly about the fillet of the die. This is linked to side effects, which can be related to the streamlines of eddy currents flowing in the sheet, as shown in Figure 11a) for a generic straight coil. Indeed, since the flat sheet is not an infinite medium (in its plane), the streamlines of eddy currents are curved, in particular close to the sheet boundaries. Provided the magnetic induction shown in Figure 2, it is then easy to show that

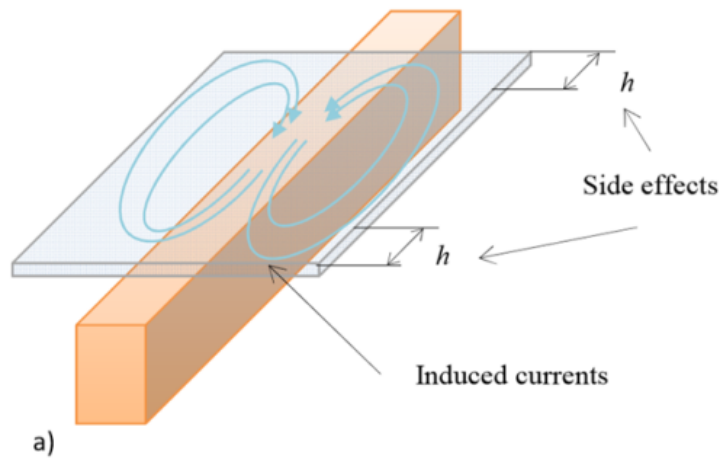

Figure 11: Side effects due to curved eddy current streamlines.

the normal component of the Lorentz forces (1) drops since the $y$-component of eddy currents $j_{y}$ now includes the cosinus of the angle defined between the direction of the coil and the local tangent to the streamlines of the flowing eddy currents. Consequently at fixed flange height (in the transverse direction), the less the flange length is, the less the Lorentz forces are. This is verified experimentally by observing that flanges of lower lengths are bent with a lower angle at constant discharged energy, as shown in Figure 11b). This phenomenon has already been observed [18], and was called the "specimen planar area" effect. 
Next, the rebound observed between the flange and the die results from waves propagation and reflexion in these two media. Tests conducted with the maximum frame rating of the camera $(675000$ fps), giving a minimal time increment between two frames of about $1.5 \mu \mathrm{s}$, allowed to conclude that the time required for the flange to rebound on the die is lower than the minimal time increment allowed by the camera. This is to be compared with the time required for a pression wave to travel forth and back through the thickness of the AA1050 flange of thickness $t=0.5 \mathrm{~mm}$, of about 0.16 $\mu s$. Therefore, the rebound is caused by the return at the flange/die interface of a rarefaction wave, due to the reflexion at the free end of the flange of the initial pressure wave generated by the impact. More precisely, the rebound results from a mismatch of material impedances between the flyer and the target. A simplified modeling describing this phenomenon is presented in Section 5.

At last, the analysis of pictures recorded from high speed cameras allows to assess an impact velocity of the flange of about $180 \mathrm{~m} \cdot \mathrm{s}^{-1}$. From the overall time of flanging $\Delta t \sim 130 \mu \mathrm{s}$, it is then possible to give an order of magnitude of strain rate in the flange close to the fillet. Assuming a

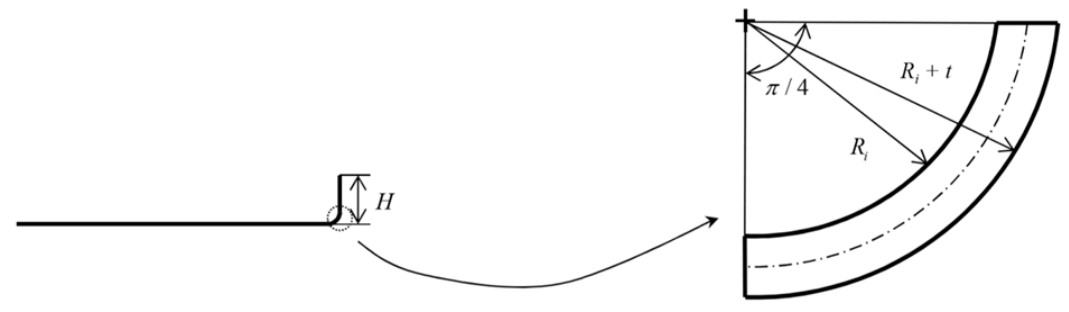

Figure 12: Fillet of the flange.

perfect kinematics of flanging at right angle as shown in Figure 12, and neglecting for the moment the formation of plastic hinges, some average strain rate during the flanging time in the fillet of the flange along its transverse direction can be assessed as follows:

$$
\dot{\varepsilon}=\frac{(\pi / 4)\left[\left(R_{i}+t\right)-\left(R_{i}+t / 2\right)\right]}{(\pi / 4)\left(R_{i}+t / 2\right)} \frac{1}{\Delta t}
$$

Thicknesses of sheets of $0.5,1$ and $2 \mathrm{~mm}$ hence yield average strain rates of about 700, 1280 and 2200 $\mathrm{s}^{-1}$ respectively. These orders of magnitude of strain rate are consistent with what is expected in the literature [3] for electromagnetic flanging.

\subsubsection{Second experiment}

Results Figure 13 shows a sequence of pictures obtained with the high speed cameras for the second experiment, in which the top and side views have been recorded at 45000 frames per seconds.

It can be observed on the top view (Figure 13(a)) that the flange is bent much more uniformly compared to the first experiment. The contact with the die occurs in picture 6 , and a rebound is still observed in picture 7. Next, the side view (Figure 13(b)) shows that the flange is essentially bent close to the fillet of the die, without triggering any additional plastic hinge. On the whole, the obtained flange does not exhibit any significant defect as previously.

To go further and in order to observe the spatial distribution of Lorentz forces generated by the optimized inductor, some tests have been conducted by placing a sheet onto a grid, the system being 


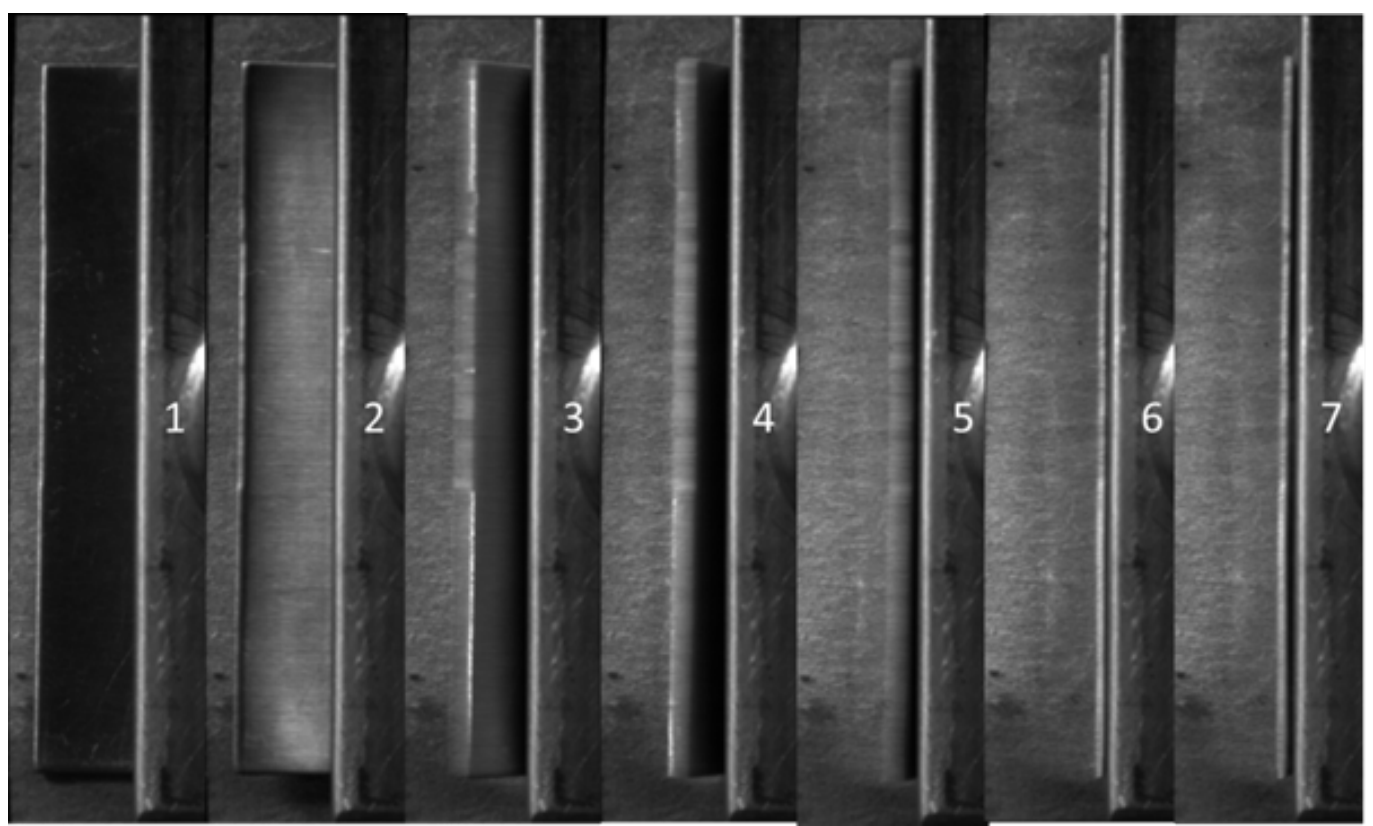

(a) Top view of the deformation process

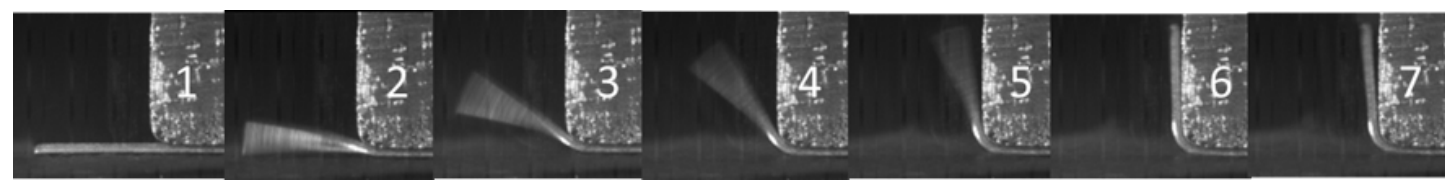

(b) Side view of the deformation process

Figure 13: Top and side views of forming a straight flange with high speed camera (45000 frames per seconds).

clamped by rigid blank holders. These tests have been conducted with a discharged energy of 3.3 $\mathrm{kV}$ and AA1050 sheets of $0.5 \mathrm{~mm}$ thickness to profit from their good compliance and ductility to improve the footprint depth for observation purpose. Figure 14 shows a comparison of the footprints generated by the grid in the sheet, with the U-shaped and the notched inductors. The more the depth of the footprint is, the more Lorentz forces are. It can be observed on the one hand the lack of footprint depth in the corners obtained with the U-shaped inductor, and on the other hand a greater one obtained with the notched inductor. A significant magnitude of Lorentz forces has been generated on the corners of the flange, close to the notches, to obtain the observed increased footprint depth, hence validating this technology.

Repeatability tests and metrological controls have been performed on the formed parts. Figure 15 shows many superposed flanges formed with both 1 and $2 \mathrm{~mm}$ sheet thicknesses made of AA2024-T4. It can be observed the good repeatability of the process. Table 4 lists the results obtained from metrological controls performed on these flanges. The average and standard deviation values of the 

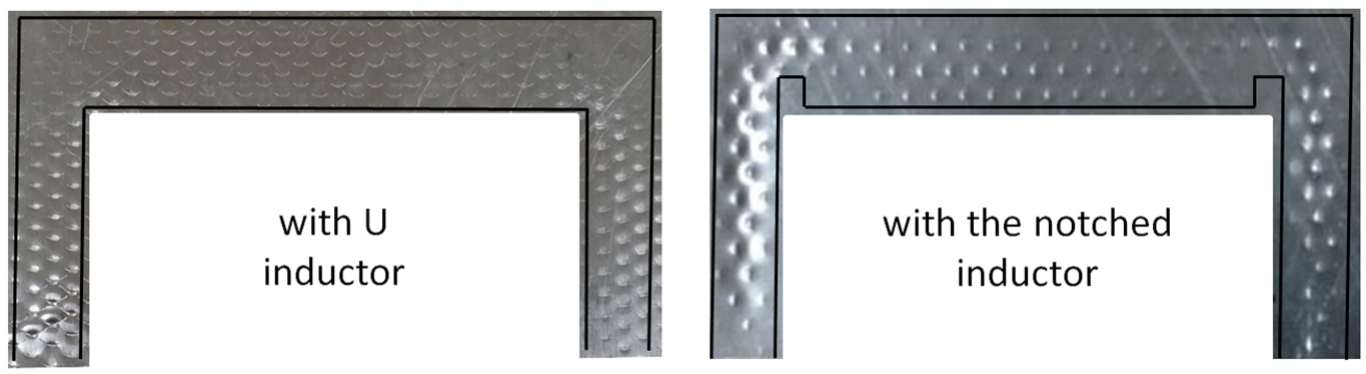

Figure 14: Grid footprints obtained with the U-shaped and the notched inductors.

straightness, the flatness, the radius of the fillet and the flanging angle are summarized for both 1 and $2 \mathrm{~mm}$ sheet thicknesses. Good tolerances are achieved, and particularly small deviations are measured. Flatness and straightness of the order of one or two tenth of millimeters are achieved for flanges of a hundred millimeters length. These tolerances thus appear consistent with an industrial process. The obtained flanging angle that is close to a right one results from the die compensation

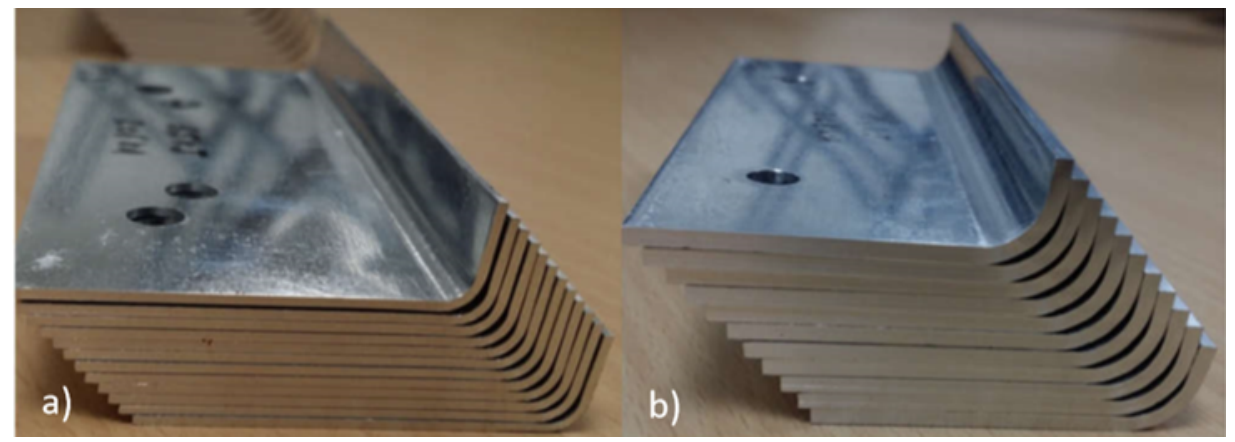

Figure 15: Repeatability experiments.

angles set to 9 and $7^{\circ}$, which have been machined in the die for sheets of 1 and $2 \mathrm{~mm}$ thicknesses respectively. Figure 16a) shows the fillet area of the flange after the deformation once polished, and Figure 16b) shows the same area after anodic oxidation. It can be observed that the grain have been deformed with dynamic straining, especially elongated in the outer area of the fillet. However, no particular damage of the material is observed, and the surface coating is not affected by the dynamic process.

Discussion The optimized inductor has been designed so that the spatial distribution of Lorentz forces in the longitudinal direction be more uniform, including the two extremities of the flange. The two notches added to the inductor (Figure 5) and placed close to the flange corners, actually play the role of corner-field shapers that aim at both concentrating eddy currents and realigning their streamlines with the coil direction at corners. This permits to avoid the drop of the normal component of Lorentz forces due to a misalignment between the streamlines of eddy currents and 


\begin{tabular}{|c|c|c|c|c|c|c|c|c|}
\hline $\begin{array}{l}\text { AA2024- } \\
\text { T4 sheet } \\
\text { thickness }\end{array}$ & \multicolumn{2}{|c|}{ Straightness (mm) } & \multicolumn{2}{|c|}{ Flatness (mm) } & \multicolumn{2}{|c|}{ Radius (mm) } & \multicolumn{2}{c|}{ Angle $\left.{ }^{\circ}\right)$} \\
\hline & $A$ & $D$ & $A$ & $D$ & $A$ & $D$ & $A$ & $D$ \\
\hline $1 \mathrm{~mm}$ & 0.253 & 0.009 & 0.157 & 0.009 & 2.912 & 0.097 & 89.884 & 0.408 \\
\hline $2 \mathrm{~mm}$ & 0.118 & 0.018 & - & - & 6.342 & 0.016 & 89.986 & 0.376 \\
\hline
\end{tabular}

Table 4: Result of metrological control of series of ten parts (straightness, flatness, bending radius and bending angle). A stands for Average, $D$ for Standard Deviation.

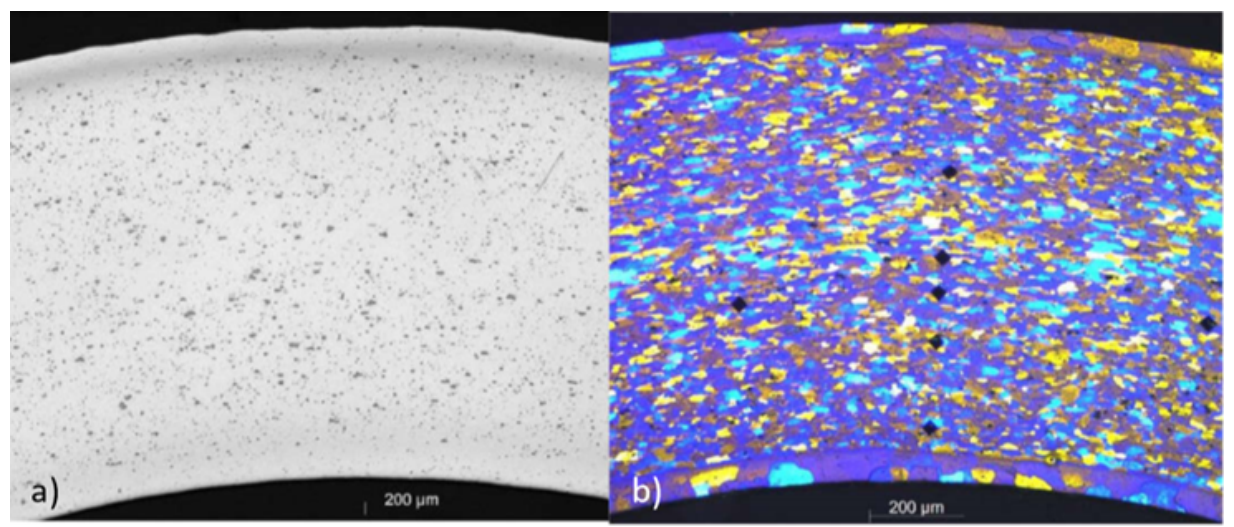

Figure 16: Metallurgical analysis after dynamic strain. a) Rough polishing on left. b) After anodic oxidation.

the longitudinal direction, and hence reduce edge effects. In addition, the cross-sectional area of the inductor is locally reduced close to edges in order to foster the flow of eddy currents in the extremities.

The plastic hinge observed on the side view in the first experiment (see Figure 10) was actually only triggered locally at the two ends of the flange, because of their delay caused by lower Lorentz forces in these areas. Three-dimensional deformation effects coupling bending in two planes were thus occurring, about both longitudinal and transverse directions. The optimized inductor allowing to remove the delay of the flange corners, these three-dimensional deformation effects are now limited, and no additional plastic hinge is observed (see Figure 13). However, it does not completely prevent the occurrence of any additional plastic hinge in electromagnetic flanging, as discussed next with the third experiment.

\subsubsection{Third experiment}

Results Figure 17 shows both the deformed shape of a flange of increased height $(20 \mathrm{~mm})$, obtained with a similar inductor to that used in the second experiment but with the same increased height, and a sequence of pictures recorded with a high speed camera in side view during the forming process. It can be observed that the flange is so much bent that an additional plastic hinge appears close to its mid-height during the forming operation (picture (ii) of Figure 17a). The flange then behaves as two 


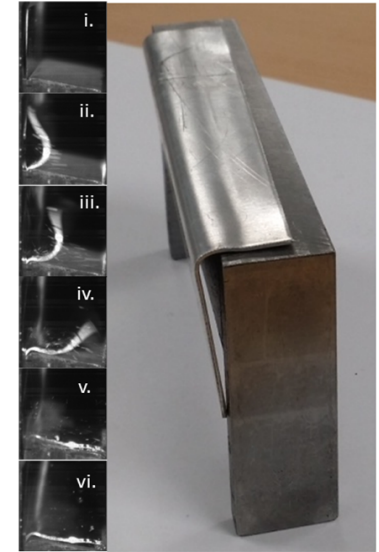

a. b.

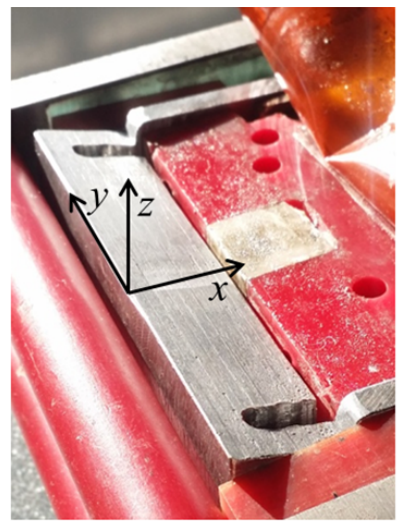

a.

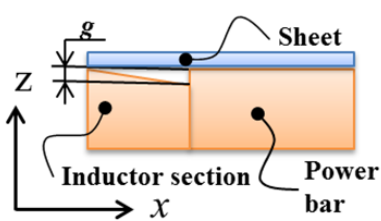

b.

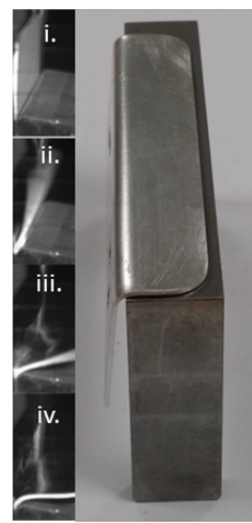

c.

Figure 17: Deformed shape of a flange of increased height and side view obtained from high Figure 18: (i) Top view of the modified inductor, (ii) sequence of pictures recorded with a high speed camera, (iii) deformed shape of the flange. speed camera.

(almost) rigid bars articulated between them and at the fillet of the die. The first bar impacts first the die (picture (iv)), followed by the second one that spans almost a right angle before hitting the die (picture $(\mathrm{v})$ ) once the first one has contacted. This phenomenon now occurs on the whole length of the flange since an almost straight fold line can be observed on the deformed shape in Figure 17b).

Discussion One way to avoid the formation of a second plastic hinge is to improve the spatial distribution of Lorentz forces in the transverse direction. Since the top area of the flange is delayed with respect to its base, Lorentz Forces have to be increased in this area so that to avoid the appearance of an additional plastic hinge. In order to implement it, two parameters can be changed. First, the thickness of the rectangular cross-section of the active area of the inductor should be decreased so that to increase the currents flowing in the skin depth. Second, a small gap should be introduced between the inductor and the workpiece in the vicinity of the edge of the metal sheet to take advantage of the decreasing magnitude of magnetic induction with the distance. These two items are shown in Figure $18 \mathrm{~b})$.

Results Figure 18c) shows a sequence of pictures recorded in side view with a high speed camera and the deformed shape of the flange. Since the cross-section of the inductor has been modified so that Lorentz forces applied on the flange decrease with the $x$-coordinate (see Figure 18b)), it is now correctly bent about the fillet of the die. The deformed shape does not exhibit any additional fold line any more, and a proper forming has been performed. 


\subsection{Curved flanges}

Figure 19a) shows a convex curved flange formed with a curved inductor, analog to that shown in Figure 5 with a constant cross-section. Significant buckling and wrinkling can be observed. Indeed, this inductor provides a quite homogeneous distribution of Lorentz forces along the curvilinear coordinate, so does hence for the impact velocity. However, since the convex geometry is a non-developable surface, a plastic flow occurs in the plane of the sheet. The edge is thus subject to compressive stresses due to the convex geometry of the die, and it yields buckling and wrinkling. In order to
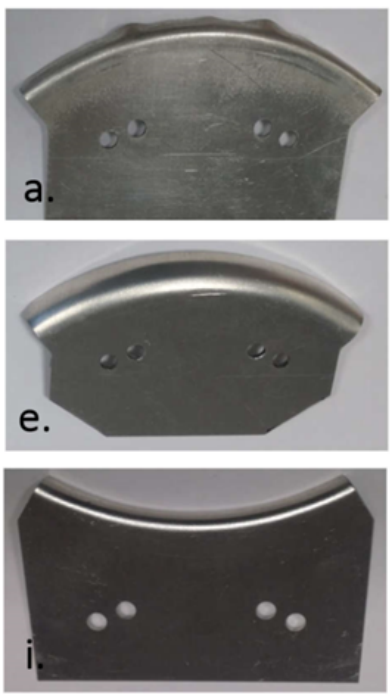
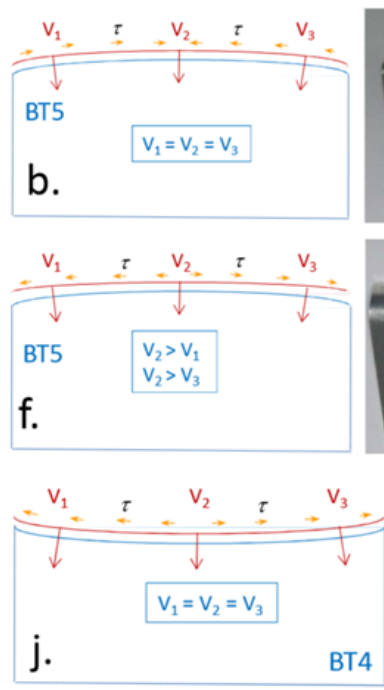
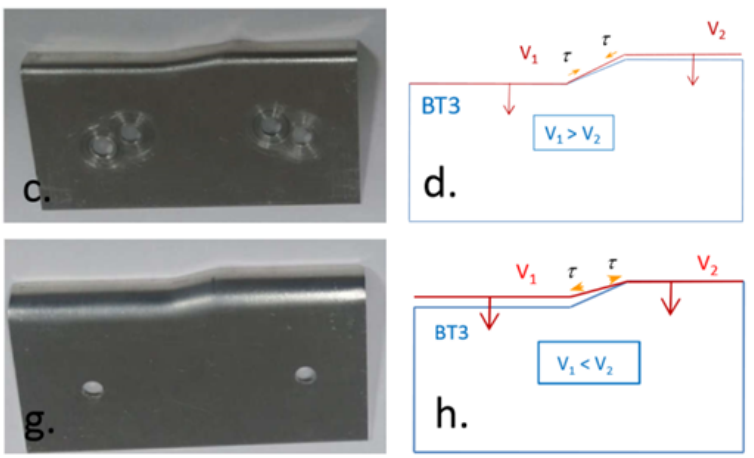

Figure 19: Convex, concave and jogged flanges.

perform a successful curved flange, a particular kinematics that consists in unrolling the flange onto the die should be respected, from the center towards the corners. To this end, the inductor should be designed in such a way that decreasing Lorentz forces are generated from the center to the extremities. Playing on the cross-section of the active area of the inductor allows to implement it. In particular, a non-constant thickness of the cross-section along the curvilinear coordinate, as shown in Figure $6 \mathrm{c})$, allows to enforce that the flange is unrolled onto the die from the center to the extremities, so that the edge is submitted to tension rather than compression. This permits to avoid wrinklings and buckling. Figure 19e) shows now a good result with that technique.

The concave geometry is not subject to compression plastic flow in the plane of the sheet as shown in Figure 19 i) and j). Good results without buckling are obtained with a curved inductor of constant cross-section.

However, the jogged flange also requires a special care. If the flange first contacts the farthest plane (thus requiring the largest bending angle), then unrolls to a closer one, a compressive area appears at their junction, as shown in Figures 19c) and 19d). Rather, starting from the closer one allows a proper forming (Figure 19g)), because only in-plane tension stresses will be generated in the junction of the two planes (Figure 19h)). More precisely, this particular kinematics of bending can be easily implemented by translating the sheet in the $x$-direction of a small gap, as shown in Figure 20 . 


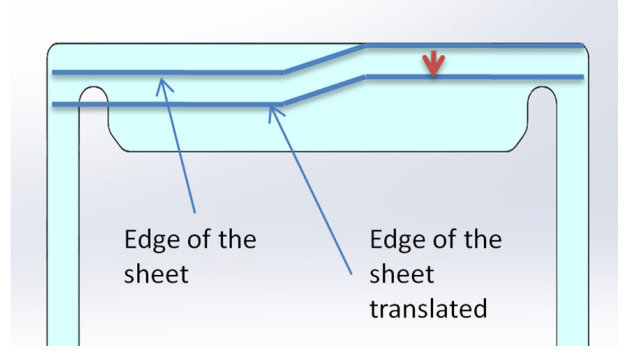

Figure 20: Relative position between the sheet and the inductor for the jogged flange.

This allows to introduce a small perturbation on the longitudinal distribution of Lorentz forces along the curvilinear coordinate of the jogged flange, so that it is properly unrolled onto the die without any compression area.

At last, Figure 21 shows the forming of a circular hole flange. Its geometry acts as a concave curved flange on a full rotational geometry. Consequently, a constant cross-section is able to correctly form this geometry, only generating tension stresses. However, input and output power bars shown in Figure 6d) have been designed such that the discharged current in these bars flow in the same direction. Indeed, a previous implementation of that inductor with parallel input and output power bars having opposite discharged current flows tended to show a lack of forming of the flange in the vicinity of these bars. This is due to a local decrease of the magnitude of the magnetic field between these bars, caused by the interaction of magnetic fields generated by currents flowing in parallel bars in the same direction. The particular design of bars shown in Figure 6d) avoids this effect, and no defect is observed on the flange close to input and output power bars of the inductor.
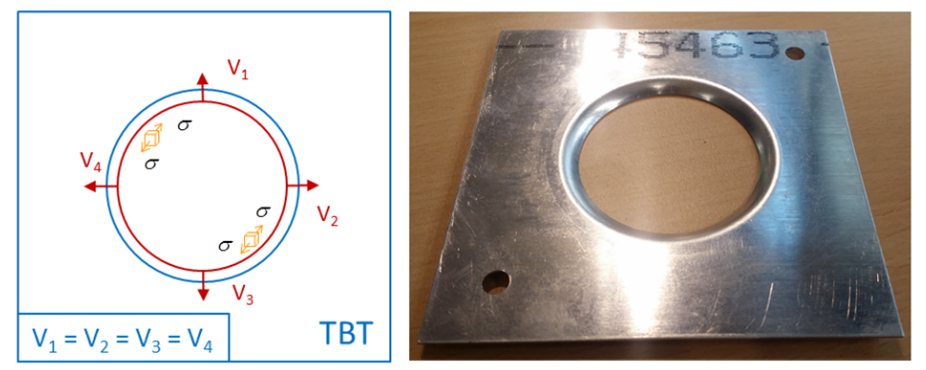

Figure 21: Circular hole flange.

Generally speaking, for some particular geometries a particular path of unrolling the flange onto the die should be respected to form a successful flange. This path should be designed in accordance with the kinematics of bending the flange, in such a way that compression areas are avoided. Other geometries (concave ones) do not require such special care to achieve a proper flange. 


\section{Simplified modeling of the rebound}

\subsection{One-dimensional considered system}

Considering for simplification a planar impact between the flange and the die. The states generated after their contact as well as the condition leading to the bounce can be described by the following simplified model, valid far from the lateral boundaries of the flange and the die. Consider two bodies $A$ and $B$, infinite in transverse directions, and of finite lengths $l_{A}$ and $l_{B}$ in the longitudinal (Lagrangian) direction respectively, as shown in Figure 22. Free boundaries are considered at extremities of the

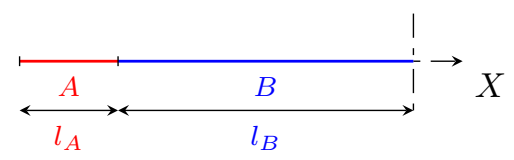

Figure 22: One-dimensional system

bodies $A$ and $B$, and initial conditions are set so that $B$ is initially in a natural state, while the body $A$ is animated of a (known) uniform velocity $\bar{u}$. The above one-dimensional model allows to answer the following questions: (i) under what conditions the body $A$ bounces on the body $B$, and (ii) what are the stress and velocity levels reached during the experiment.

\subsection{Hydrodynamic approximation}

The above problem can be first treated using the so-called hydrodynamic approximation, which consists in considering the solid as an inviscid compressible fluid, thus neglecting the shearing strength of solids. Such approximation is usually employed for solids undergoing high levels of pressure. From the conservation laws of mass, momentum and energy, the Rankine-Hugoniot conditions associated to any shock front can be written, here in Lagrange variables [21]:

$$
\begin{aligned}
& {[u]=\mp \rho_{0} \mathscr{D}[V]} \\
& {[p]= \pm \rho_{0} \mathscr{D}[u]} \\
& {[E]=\mp \frac{1}{2}\left(p^{-}+p^{+}\right)[V]}
\end{aligned}
$$

where $u, V, p, E$ and $\mathscr{D}$ stand for the material velocity, the specific volume, the pressure, the specific internal energy and the material wave velocity respectively, while $[\cdot]=(\cdot)^{+}-(\cdot)^{-}$denotes the jump of the quantity $(\cdot)$ across the shock front. Weighting signs \pm are such that the top and bottom ones pertain to rightward and leftward waves respectively.

Following [21], the results shown in Figure 23, here extended to the hydrodynamic approximation, can be recalled. Figure 23(a) shows the characteristics in the $(X, t)$ plane and the mechanical states in the $(u, p)$ plane in the case where a material of low impedance impacts a material of high impedance, such that $Z_{A} / Z_{B}<1, Z_{i}=\rho_{0_{i}} \mathscr{D}_{i}, i=A, B$, denoting the material impedance. The mechanical states are determined by means of the Rankine-Hugoniot condition (3), or analogously by the matching impedance technique [22]. The impact between the bodies $A$ and $B$ generates two rightward and leftward shock waves in the bodies $A$ and $B$ respectively, of shocked state 2 . The reflexion of the leftward shock wave on the left free boundary yields an unloading rarefaction wave propagating 
rightward. For nonporous solid materials and if the pressure is not that high, both the Hugoniot curve of a shock wave and the isentropic curve of a rarefaction wave are very close, hence the isentropic curve can be used as an approximation for the unloading path [21]. Since the material impedance of the body $A$ is lower than that of $B$, the unloading process gives the state 3 that consists of a zero pressure and a negative velocity. When the unloading reflexion reaches the interface, the body $A$ rebounds with velocity $v_{3}$, while an unloading pressure pulse still propagate in $B$.
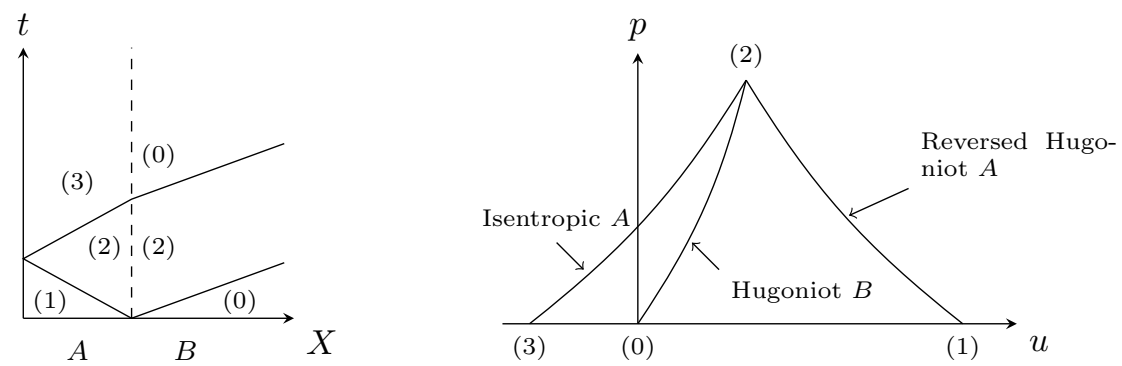

(a) $\frac{Z_{A}}{Z_{B}}<1$
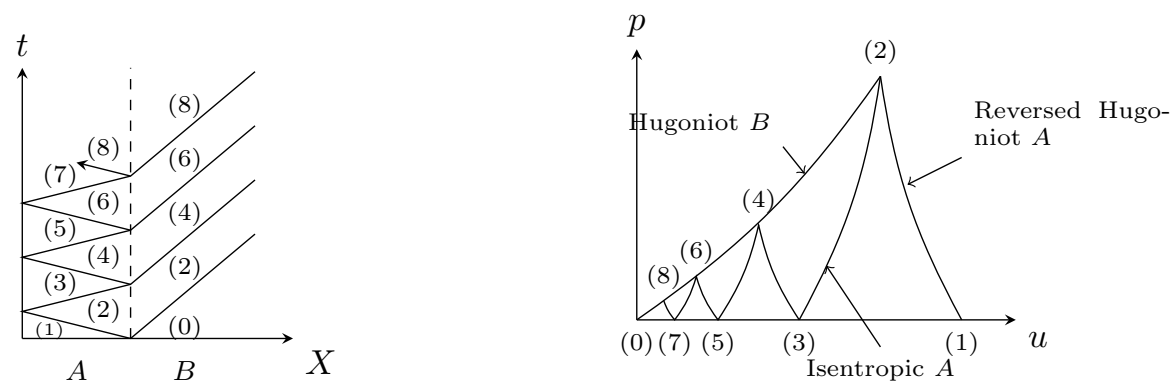

(b) $\frac{Z_{A}}{Z_{B}}>1$

Figure 23: Impact between the flange and the die. $Z_{i}=\rho_{0_{i}} \mathscr{D}_{i}, i=A, B$, denotes the material impedance.

Conversely, if a material of high impedance impacts a material of low impedance, such that $Z_{A} / Z_{B}>1$ (see Figure 23(b)), then the unloading wave reflected at the free boundary of $A$ yields now a positive velocity (state 3 ). It means that the collision has not ended yet, and as the reflected unloading wave contacts the interface, it is both transmitted and reflected again, hence unloading the previous shocked state from 2 to 4 . Several round trips of waves are required to completely unload the interface between the two bodies, but no rebound now occurs. A particular case of the two former ones is obtained for equal material impedances of the two impacted parts, for which the first reflected unloading wave brings the velocity in state 3 directly back to zero, so that no rebound should occur as well.

Consider now the second case $\left(Z_{A} / Z_{B}>1\right)$, for which the even numbers of states (2-4-6-8) transmitted in the body $B$ then reach the right free boundary of $B$ (at coordinate $X=l_{A}+l_{B}$ ) and are reflected. Figure 24 shows the characteristics plane and the mechanical states associated with this process of reflexion. 

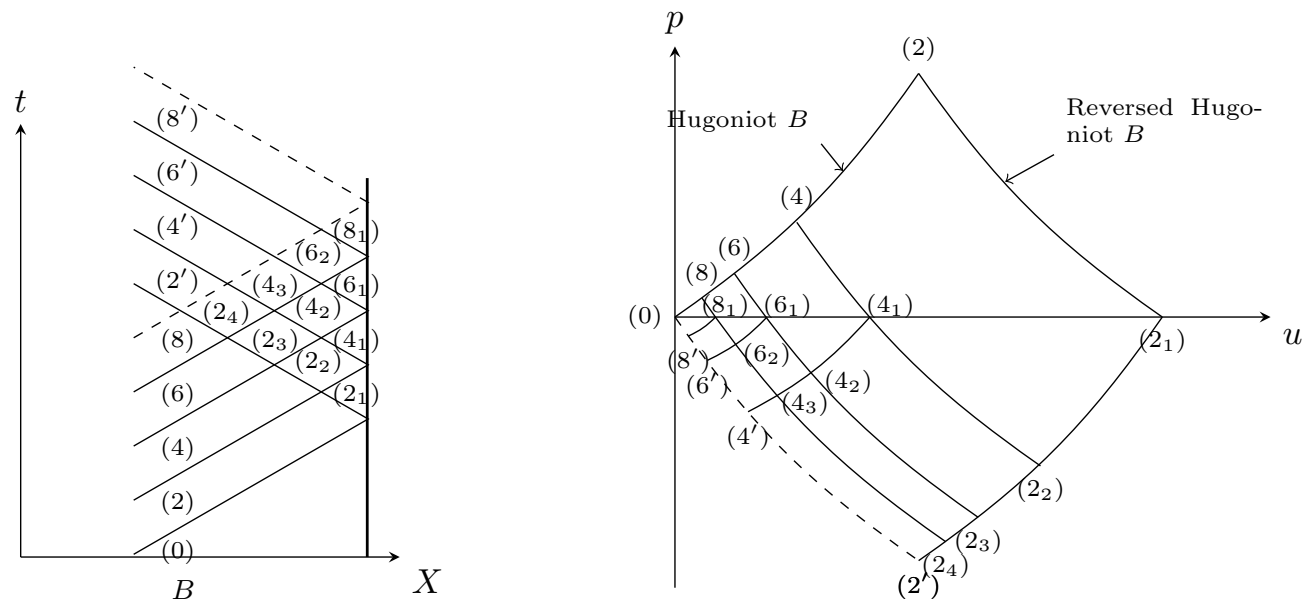

Figure 24: Reflexion at the right free end of body $B$ of the transmitted pulses.
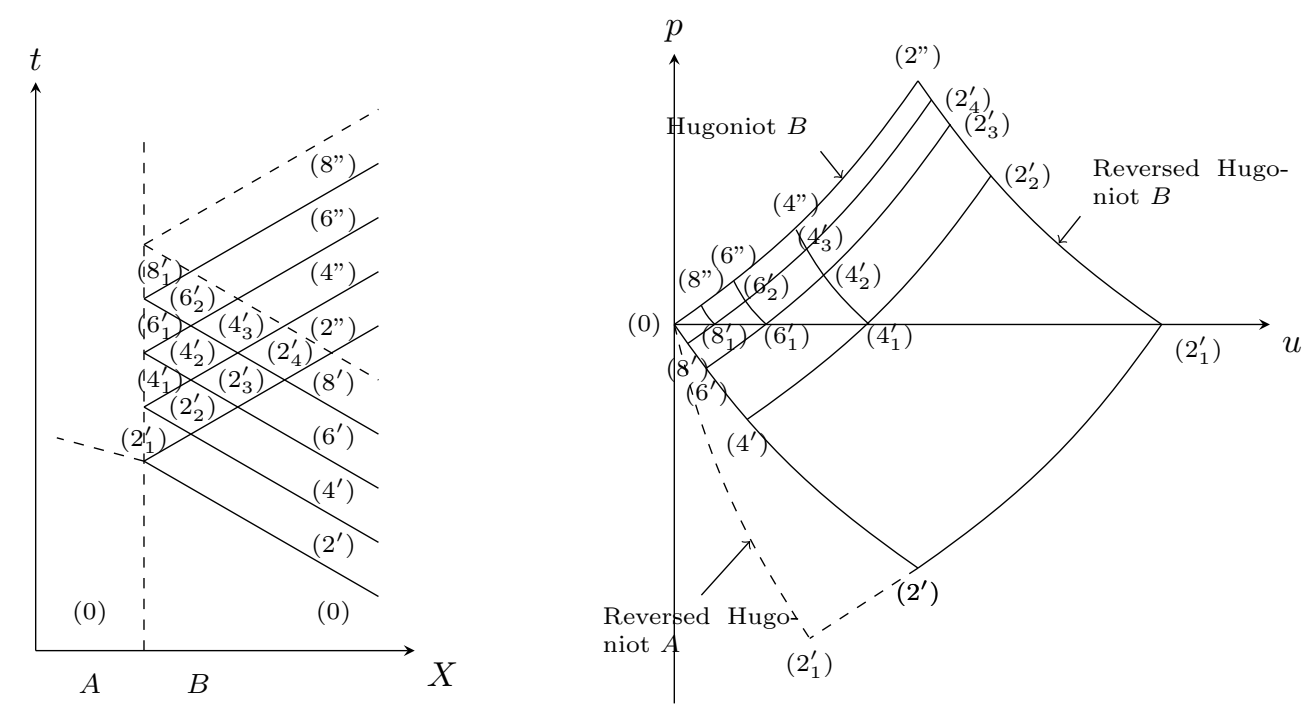

Figure 25: Return of tensile waves at the interface between the bodies $A$ and $B$.

The pressure waves generated from the impact between the two bodies are reflected at the right free end of $B$ as tensile waves propagating leftward, after a set of intermediate reflexions as shown in Figure 23. Everything happens as if the input states are reflected as states with the same velocity and with opposite (tension) pressure. These tensile waves are known to sometimes generate spallation. However, assuming the tensile strength of $B$ is sufficient to withstand these states, the tensile waves now propagates leftward until they reach the interface between the bodies $A$ and $B$, which is at 
that time in a natural state. Figure 25 shows the characteristics plane and the mechanical states associated with the return of the tensile waves at the interface. If the bodies $A$ and $B$ were stuck, the state $2_{1}^{\prime}$ would consists of a higher tension stress and a lower velocity with respect to state $2^{\prime}$ (plotted in dashed lines in Figure 25). But here, unilateral contact conditions have to be considered at the interface, which cannot withstand any tension stress. Hence, the interface between the bodies $A$ and $B$ actually behaves as a free boundary: the stress drops to zero and the velocity is doubled, while the body $A$ remains at rest. Everything happens as if the body $B$ was bouncing on the body $A$. The states associated with the rightward propagating reflected waves are identical to initial ones generated by the impact. The contact between the two bodies is again lost because the transmitted pressure waves have been reflected into tensile ones via the right free boundary.

\subsection{Linearization}

High speed cameras allowed to assess the impact velocity to be of the order of 180 m.s ${ }^{-1}$ in the first experiment. Provided the flange and the die respectively made of aluminium 1050 and of a basic steel, the pressure associated with the state 2 shown in Figure 23(a) is slightly less than 2 GPa. These relatively 'low' levels of pressure allow to simplify the above modeling by linearizing the set of equations. Indeed, the Hugoniot curves associated with the hydrodynamics approximation can be replaced by straight lines, which amounts to consider linear elastic constitutive models for the media $A$ and $B$. Therefore, the propagation speed of waves $\mathscr{D}$ is considered constant and equals that of pressure waves in this type of medium $c_{L}=\sqrt{(\lambda+2 \mu) / \rho}$, where $\lambda$ and $\mu$ denote Lamé's parameters. The Rankine-Hugoniot conditions (2)-(4) can be rewritten replacing the pressure $p$ by the opposite of the longitudinal stress $-\sigma$, the variation of the specific volume $\left(V_{0}-V\right) / V_{0}$ by the longitudinal strain $\varepsilon$, and the material wave velocity $\mathscr{D}$ by the pressure wave velocity $c_{L}$. The errors made on the pressure and the velocity computed with the above data are of the order of $2 \%$, which is neglectable with regards to the present objectives. The interest of electromagnetic flanging with respect to explosive forming lies in that lower levels of pressure are achieved, while it still profits from dynamical effects.

\subsection{Summary}

Whatever the material impedance ratio between bodies $A$ and $B$, this small model predicts that a rebound will occur after the impact: after one round-trip of wave in the body $A$ if $Z_{A}<Z_{B}$, or after that the transmitted pressure waves to the body $B$ have been reflected into tensile ones via the right free boundary if $Z_{A}>Z_{B}$. From a practical viewpoint, the rebound is solved for the moment with a die compensation angle as explained in Sections 2 and 4.

\section{Case study of a model aeronautical part}

\subsection{Experimental results and discussion}

Results Figure 26 shows the deformed shapes of two parts made of AA2024-T4 and AA1050. Several observations can be made. First, the circular hole flange has been correctly formed. Second, several significant defects are observed. The longest straight flange does not have as good straightness and flatness as these measured during the second experiment (for a sole straight flange). The same also holds true for the smaller straight flange. Next, the jogged flange shows some wrinkles at the 


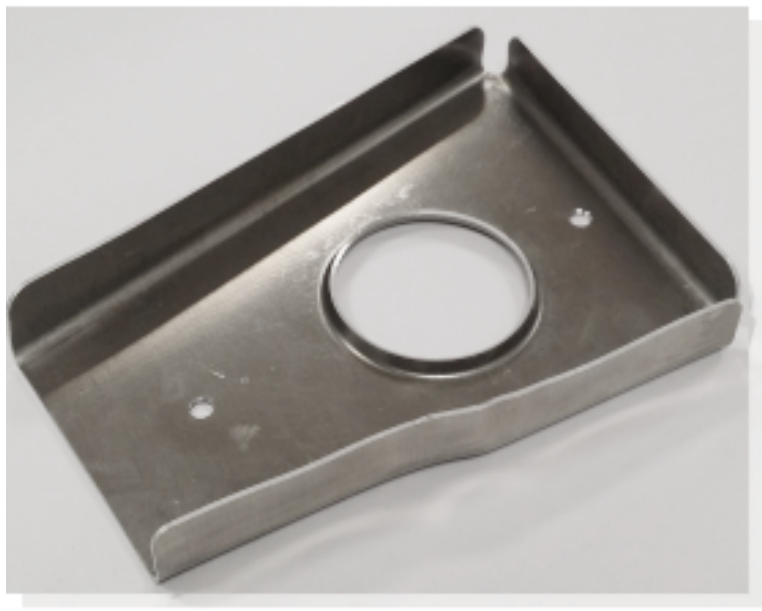

(a) Isometric view of a AA2024-T4 formed part

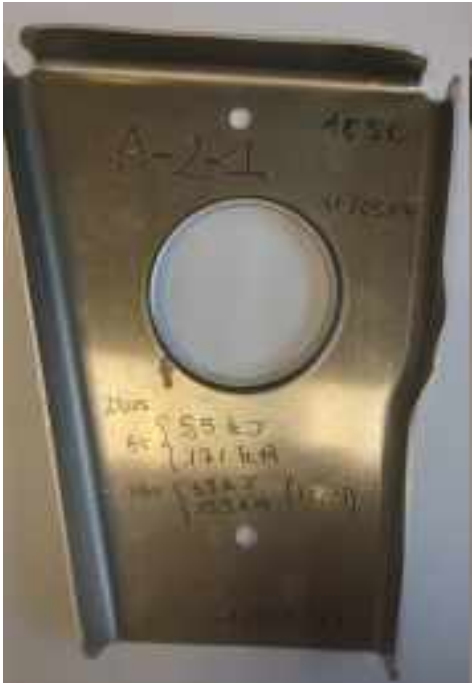

(b) Top view of a AA1050 formed part

Figure 26: Deformed shape

junction between its two planes. In particular, the AA1050 allows to better observe it due to its high compliance.

Discussion No apparent defect is observed for the circular hole flange, which has been formed on his own during the first step. Consistent results are obtained with respect to single curved flanges discussed in Section 4.2. Defects are only observed when several elementary geometries are formed simultaneously. These defects may be due to the existence of some spurious electromagnetical interactions during the discharge between different areas of the coil. In particular, the wrinkles observed on the jogged flange are due to local buckling, meaning that the flange has not been unrolled from the closest plane to the farthest one as expected, but rather the converse has occurred.

These spurious electromagnetical interactions are not trivial to understand directly. To this end, the numerical simulation appears as one tool allowing on the one hand to reproduce the experiment numerically, and on the other hand to access fields that can permit to better understand these physical phenomena.

\subsection{Numerical modeling}

The system considered consists of the part, two coils (one for the circular hole, and one for the lateral flanges), the die and a support. A volumic (solid) modeling is considered for the part and the inductors, which are meshed with solid HEXA8 elements. The sole part is considered as deformable, the coils are rigid and fixed. The die and the support have been modeled as electrically insulating fixed rigid shells, and meshed with QUAD4 elements. Five elements in the thickness of the part have been used, so that the complete model approximately consists of 48000 elements. Meshes of the part and the die are shown in Figure 27. Unilateral contact conditions are considered between the part and other elements of the system. Besides, the constitutive response of the AA2024-T4 is modelled 


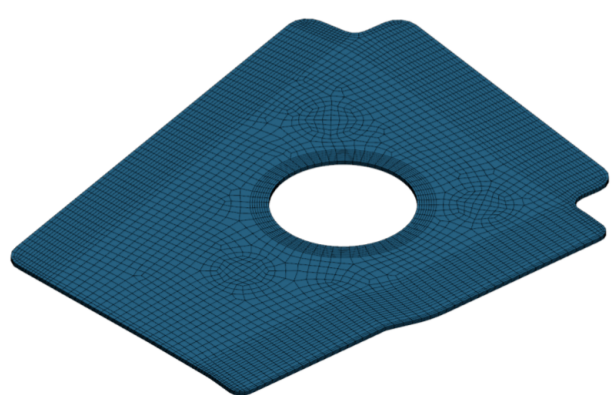

(a) Part

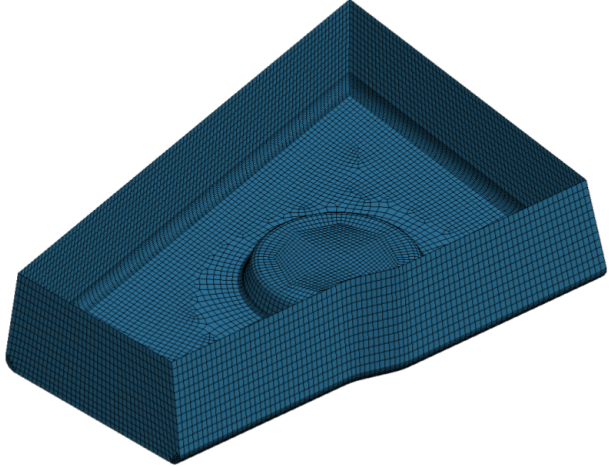

(b) Die

Figure 27: Meshes

with a rate-independent elastic-plastic constitutive model with a nonlinear isotropic hardening fitted on experimental data, because this alloy is known to be quite rate-insensitive within the regime of interest [23]. The electrical discharge is classically modeled by a RLC circuit, which provides a damped sinusoidal profile of the intensity time evolution. The solution process is conducted with the software LS-DYNA [8], that couples thermal, electromagnetical and mechanical effects. As for the experiment, the simulation is run in two steps: the first one consists in forming the circular hole flange with the first coil, the second forms the lateral flanges simultaneously with the second coil after the transfer of the numerical fields obtained and the end of the first step as initial data. To save computation time, the electromagnetic solver is stopped after almost two pseudo-periods of discharge of the current for each step, because electromotive Lorentz forces are then considered neglectable. The computation of the first step lasts about 3.7 hours on 28 CPUs for a simulation time of $0.1 \mathrm{~ms}$, while that of the second one lasts about 16 hours for $2 \mathrm{~ms}$ of simulation time.

\subsection{Numerical results}

Figure 28 shows some isovalues at the end of the bending of flanges. As expected, the plastic strain (see Figure 28(a)) is high close to the bending fillets, but it reaches its maximum at the corners between the lateral flanges. Mises stresses (see Figure 28(b)) show the same pattern, the location of the stress peak being shown in a zoom of the rear side of two extremities of two neighbor flanges. Figure 29 show the eddy currents flowing in the part at a time close to that of the first peak of the discharged current. It can be observed that their distribution is quite heterogenous, some concentration occurring at the corners of flanges. As a consequence, a high value of Lorentz forces is computed in these areas, as shown in Figure 30(a). It can be expected that these high concentrations play a major role in the final deformed shape computed. Later and once the flanges have started to be bent (see Figure 30(b)), these forces dramatically drop (four order of magnitude difference). The inertia then permit to finish to bend the flanges. 


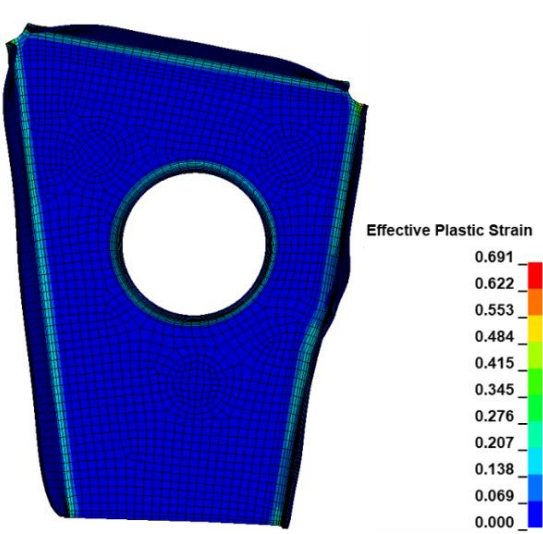

(a) Effective plastic strains (top view)

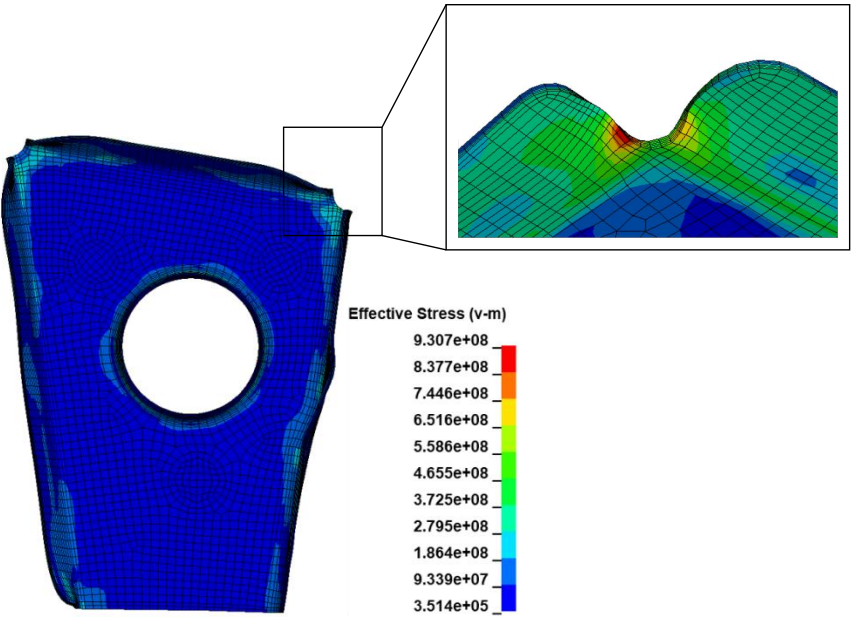

(b) Mises norm of stresses (Top view). Zoom from the bottom view of the corner.

Figure 28: Isovalues of fields in the part.

\subsection{Comparison between experimental and numerical results}

The comparison between the experimental and numerical results here essentially focuses on the respective final deformed shapes. For comparison purpose, the experimental deformed shape has been digitized by scanning 3D. Next a surface mesh is built from the measured cloud of points. Figure 31 shows several views of a superposition of the digitized experimental deformed shape (in red) and the computed deformed shape (in blue). A correct qualitative superposition between both deformed shapes is observed. However, differences exist that can be more precisely appreciated on each flange by zooming, as shown in Figure 32. First, a very good agreement is observed for the circular hole flange, though some slight differences on the bending curvature can be noticed. Second, significant differences are observed between the experiment and the numerical simulation for the straight flanges. Significant defect of straightness, flatness and perpendicularity of the straight flanges is predicted by the simulation, more than these measured on the experimental part, although it also contains defects. In particular, the corners of these flanges are bent in the simulation, which is not observed experimentally. This may be due to the high local concentration of Lorentz forces in these areas observed in Figure 30(a). A better agreement is observed on the jogged flange. The defect of wrinkling is correctly captured by the numerical simulation.

Overall, first results obtained from the numerical simulation are encouraging, although the numerical modeling should be improved to better fit experimental data. Next, forming complex parts combining several elementary geometries of flange requires to control the distribution of Lorentz forces in such a way that (i) electromagnetical interactions between different areas of the coil be accounted for, and (ii) the basic rules to achieve correct straight and curved flanges discussed in Section 4 be respected. The design of the geometry of an inductor generating such desired spatial distribution of Lorentz forces may hence be the solution of a shape optimization problem. To this end, mesh morphing tools are of great interest to fulfill this goal, and form a perspective to this work. 


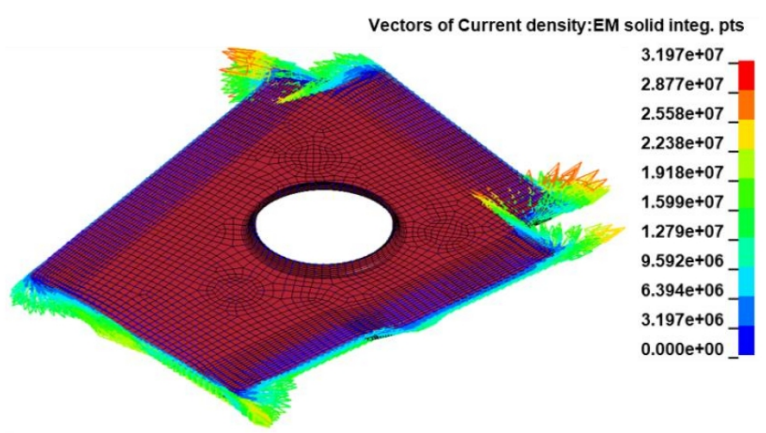

Figure 29: Eddy currents at time $t=7.5 \times 10^{-3} \mathrm{~ms}$.

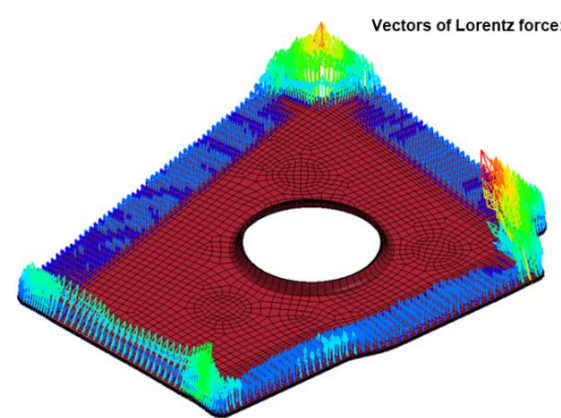

(a) $t=7.5 \times 10^{-3} \mathrm{~ms}$

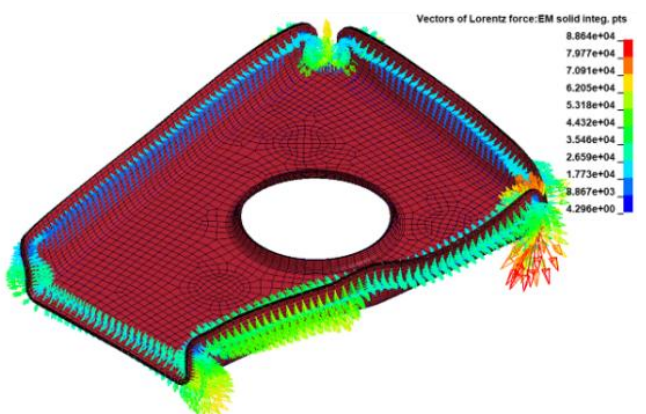

(b) $t=0.15 \mathrm{~ms}$

Figure 30: Lorentz forces at two different times.

\section{Conclusion}

A set of experiments of electromagnetic flanging performed on elementary geometries and on a model aeronautical part has been described in this paper. Elementary geometries have allowed to observe the formation of some geometrical defects that may occur during the forming of flanges, either by means of high speed cameras or just with the deformed shape. Four issues have been addressed in this paper: (i) the so-called "edge effect", (ii) the formation of plastic hinges in the flange, (iii) the rebound between the flange and the die, and (iv) the formation of wrinkles in convex or jogged flanges.

The first one occurs due to a misalignment between the streamlines of eddy currents and the longitudinal direction of the flange at their corners, and also more generally to a lack of eddy currents at these corners (eddy currents actually tend to flow along the shortest path that minimizes the spent energy). This is solved by machining notches in the inductor close to flange corners to force the realignment of eddy currents and increase their density.

The second one is due to too much bending of the flange and too few Lorentz forces applied at the top of the flange. This is solved by playing on the geometry of the cross-section of the inductor and/or on its $z$ position, so that a greater force magnitude is applied on the flange top area in order to avoid the formation of an additional plastic hinge. 

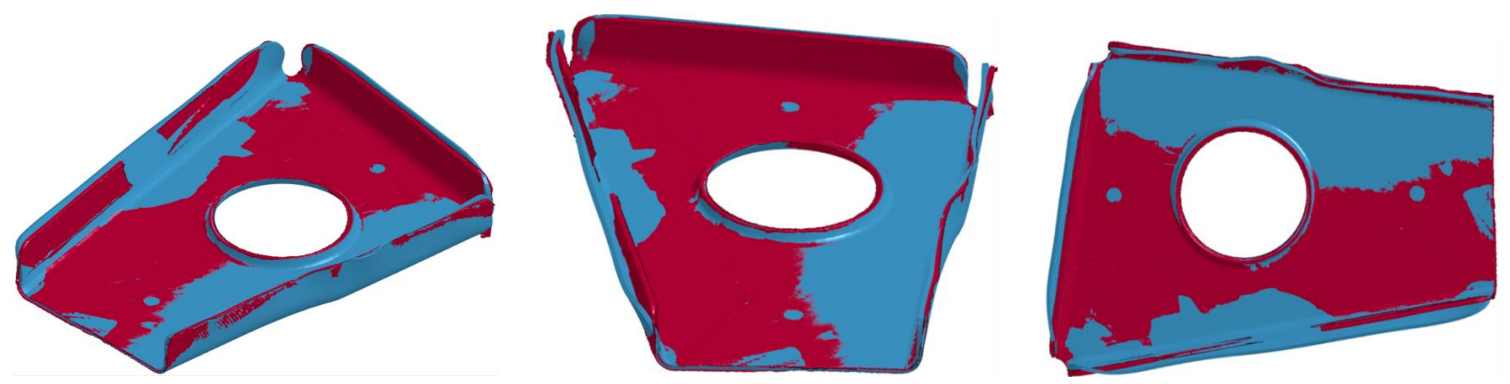

Figure 31: Superposition of the digitized experimental deformed shape (in red) and the computed deformed shape (in blue).

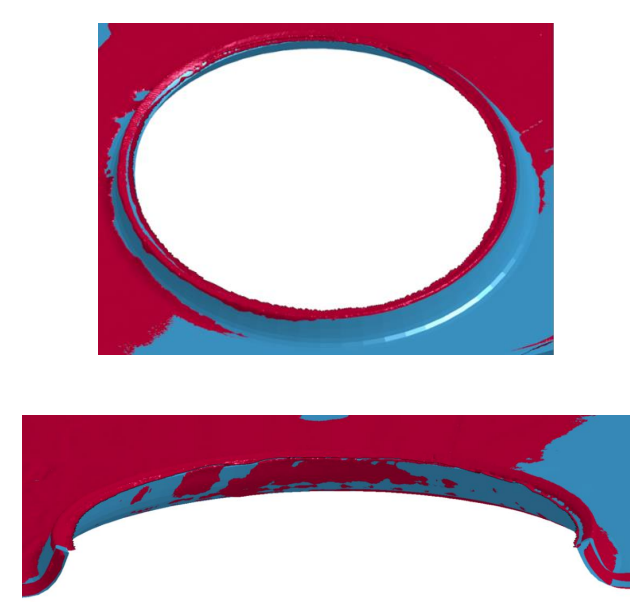

(a) Circular hole flange

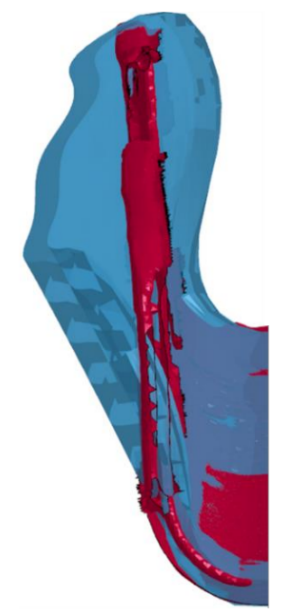

(b) Long straight flange

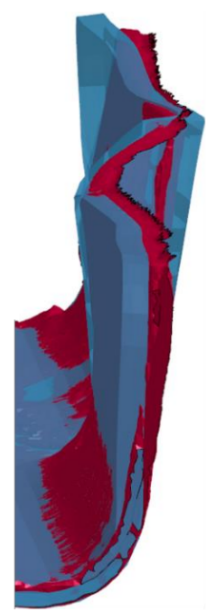

(c) Jogged flange

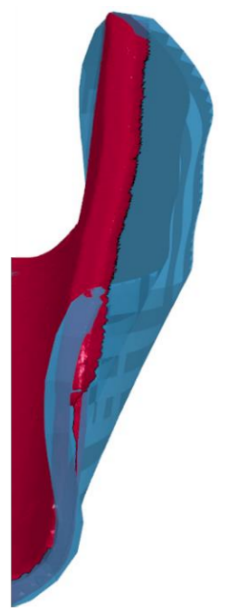

(d) Short straight flange

Figure 32: Superposition for particular flanges of the digitized experimental deformed shape (in red) and the computed deformed shape (in blue).

The third one is due to an unfavorable material impedance ratio between the flange and the die. However, a favorable material impedance ratio would also lead to bouncing, not after one round-trip of pressure waves in the thickness of the flange, but after the pressures waves generated in the die, then reflected as tensile ones at the right free boundary, get back to the flange/die interface. The best solution would be to make sure the pressure waves do not get back to the interface. This issue is solved for the moment with a die compensation angle.

The fourth and last one occurs when the curved flange undergoes locally compression in its plane, which leads to wrinkling and buckling. This is solved by designing a particular path of unrolling the flange onto the die so that no area of compression occurs.

Provided the above issues are addressed, it has been shown that it is possible to produce flanges with elementary geometries which are safe and repeatable with small variations. However, the combination of many elementary geometries required to form a model part has been shown to raise 
some challenges, especially due to electromagnetic interactions between eddy currents in the different subareas of the part and the coil. Defects can appear that were not observed in each elementary experiment. The numerical simulation then appears as a possible mean to better understand these interactions. The conducted simulations have shown their ability to reproduce the experimental deformed shape at least qualitatively. However, big differences between both are still observed especially for the straight flanges. To this end, the numerical modeling should be improved so that numerical and experimental deformed shapes better match. The next step pertains to the optimization of the geometry of the inductor, that should lead to a conform manufactured part. The use of shape optimization tools hence forms a perspective to the present work.

\section{Acknowledgements}

This study is part of the HPP project managed by IRT Jules Vernes (French Institute in Research and Technology in advanced manufacturing Technologies for composite, metallic and hybrid structures). The authors wish to acknowledge the industrial and academic partners of the project: Airbus, Airbus Group Innovations, Constellium, Europe Technologies, Stelia Aerospace and Ecole Centrale de Nantes respectively. The authors also wish to acknowledge DynaS+ for the simulation performed on LSDYNA.

\section{Conflict of interest}

The authors declare that they have no conflict of interest.

\section{References}

[1] I. Karaagac, Arabian Journal for Science and Engineering 42(5), 1853 (2017)

[2] M. Seth, V.J. Vohnout, G.S. Daehn, Journal of Materials Processing Technology 168(3), 390 (2005)

[3] V. Psyk, D. Risch, B.L. Kinsey, A.E. Tekkaya, M. Kleiner, Journal of Materials Processing Technology 211(5), 787 (2011)

[4] U. Lindholm, R. Bessey, G. Smith, J MATER 6(1), 119 (1971)

[5] V.S. Balanethiram, G.S. Daehn, Scripta Metallurgica et Materialia 30(4) (1994)

[6] S.F. Golovashchenko, Journal of materials engineering and performance 14(4), 508 (2005)

[7] B. Avitzur, Metal forming: processes and analysis, vol. 968 (McGraw-Hill New York, 1968)

[8] P. L' Eplattenier, G. Cook, C. Ashcraft, M. Burger, J. Imbert, M. Worswick, steel research international 80(5), 351 (2009)

[9] Z. Lai, X. Han, Q. Cao, L. Qiu, Z. Zhou, L. Li, IEEE transactions on Applied Superconductivity 24(3), 1 (2013)

[10] H. Su, L. Huang, J. Li, G. Li, P. Huang, Procedia engineering 207, 335 (2017) 
[11] H. Yu, Q. Zheng, S. Wang, Y. Wang, Journal of Materials Processing Technology 257, 54 (2018)

[12] Q. Xiong, H. Huang, L. Xia, H. Tang, L. Qiu, The International Journal of Advanced Manufacturing Technology pp. 1-8 (2019)

[13] W. Luo, L. Huang, J. Li, X. Liu, Z. Wang, Journal of Materials Processing Technology 214(11), $2811(2014)$

[14] H. Su, L. Huang, J. Li, F. Ma, P. Huang, F. Feng, International Journal of Machine Tools and Manufacture 124, 99 (2018)

[15] P. Jimbert, I. Eguia, M. Gutierrez, B. Gonzalez, G. Daehn, Y. Zhang, R. Anderson, H. Sundberg, S. Olsson, P. Brännström, in Proceedings of the 3rd International Conference on High Speed Forming - ICHSF (Citeseer, 2008), pp. 163-172

[16] J. Shang, P. L'Eplattenier, L. Wilkerson, S. Hatkevich, in 11th International LS-DYNA Users Conference, Detroit (2010), pp. 10-27

[17] R. VanBenthysen, B. Kinsey, HIGH SPEED FORMING 2010 p. 208 (2010)

[18] R. VanBenthysen, E. Thibaudeau, B.L. Kinsey, Journal of Manufacturing Processes 15(2), 194 (2013)

[19] H.G. Noh, W.J. Song, B.S. Kang, J. Kim, Journal of Mechanical Science and Technology 28(8), $3263(2014)$

[20] X. Liu, L. Huang, H. Su, F. Ma, J. Li, Materials 11(8), 1450 (2018)

[21] L. Wang, Foundations of stress waves (Elsevier, 2011)

[22] M.A. Meyers, Dynamic behavior of materials (John wiley \& sons, 1994)

[23] J. Hodowany, G. Ravichandran, A. Rosakis, P. Rosakis, Experimental mechanics 40(2), 113 (2000) 